
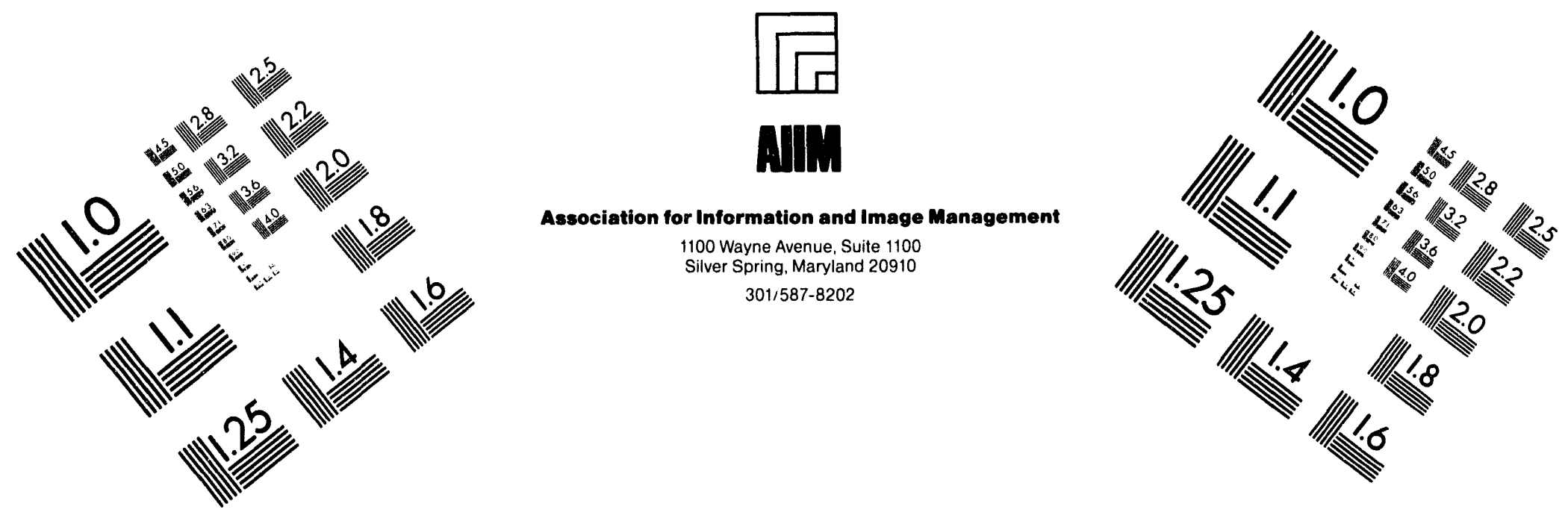

\title{
Centimeter
}

$\begin{array}{llllllllllllllll}1 & 2 & 3 & 4 & 5 & 6 & 7 & 8 & 9 & 10 & 11 & 12 & 13 & 14 & 15 & \mathrm{~mm}\end{array}$

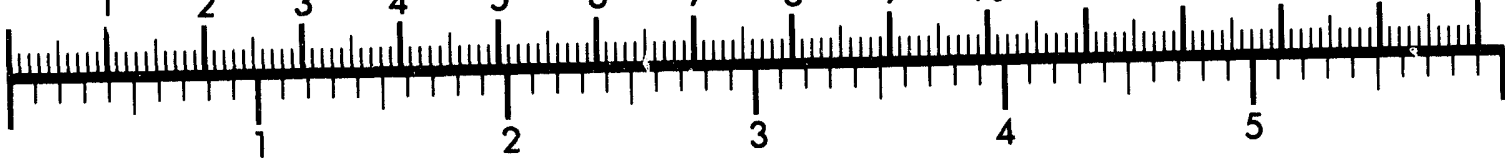
Inches
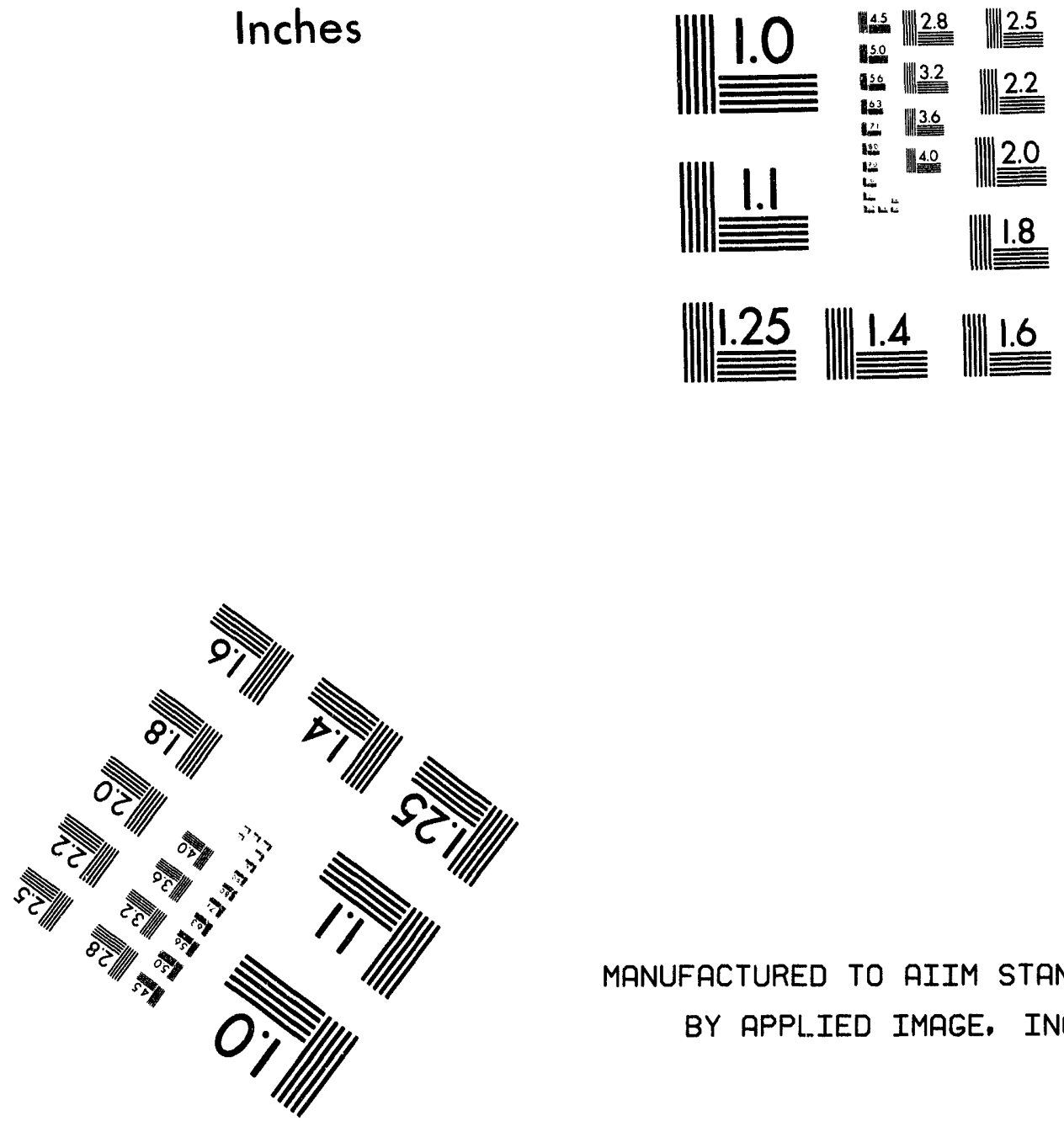

MANUFACTURED TO AIIM STANDARDS

BY APPLIED IMAGE, INC.

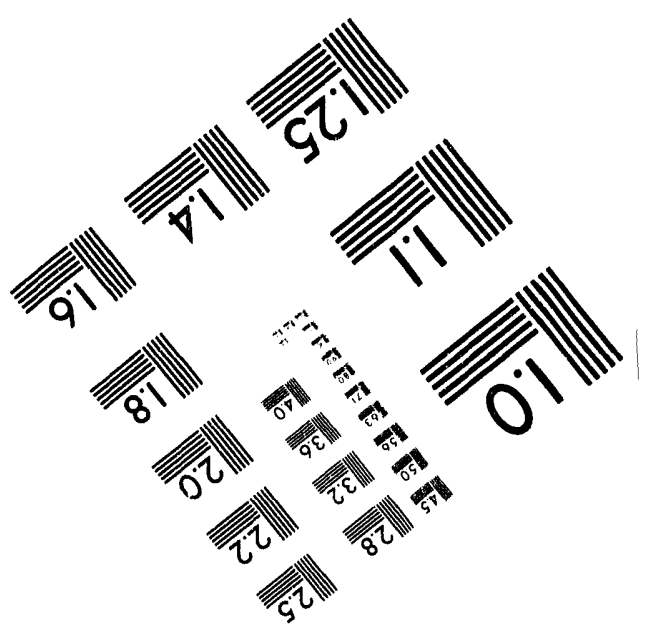



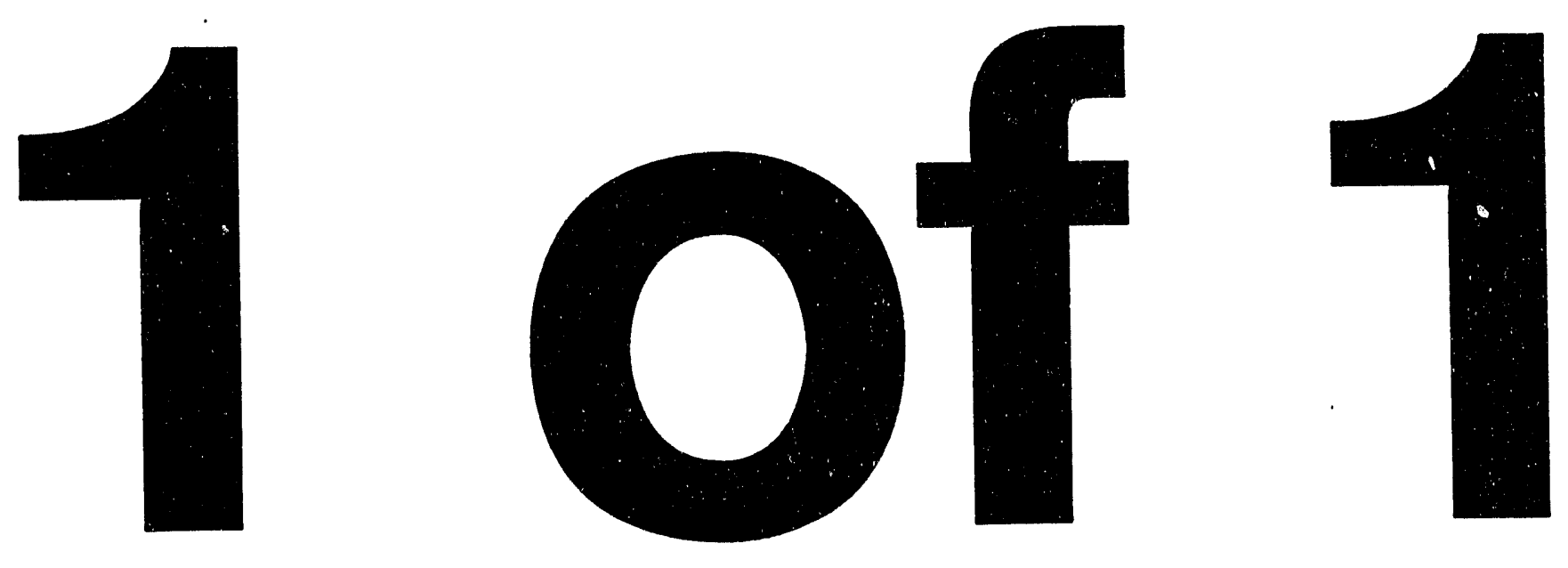
SAND94-0320 - UC -700

Unlimited Release

Printed May 1994

\section{Analysis of Anelastic Flow and Numerical Treatment via Finite Elements}

M. J. Martinez

Propared by

Sandia National Laboratorloe

Albuquerque, Now Mexico 87185 and Livermore, Callfornis 94550

for the United States Department of Energy

under Contract DE-ACO4-04AL85000

Approved for public release; distribution is unlimited. 
Issued by Sandia National Laboratories, operated for the United States Department of Energy by Sandia Corporation.

NOTICE: This report was prepared as an account of work sponsored by an agency of the United States Government. Neither the United States Government nor any agency thereof, nor any of their employees, nor any of their contractors, subcontractors, or their employees, makes any warranty, express or implied, or assumes any legal liability or responsibility for the accuracy, completeness, or usefulness of any information, apparatus, product, or process disclosed, or represents that its use would not infringe privately owned rights. Reference herein to any specific commercial product, process, or service by trade name, trademark, manufacturer, or otherwise, does not necessarily constitute or imply its endorsement, recommendation, or favoring by the United States Government, any agency thereof or any of their contractors or subcontractors. The views and opinions expressed herein do not necessarily state or reflect those of the United States Government, any agency thereof or any of their contractors.

Printed in the United States of America. This report has been reproduced directly from the best available copy.

Available to DOE and DOE contractors from

Office of Scientific and Technical Information

PO Box 62

Oak Ridge, TN 37831

Prices available from (615) 576-8401, FTS 626-8401

Available to the public from

National Technical Information Service

US Department of Commerce

5285 Port Royal Rd

Springfield, VA 22161

NTIS price codes

Printed copy: A03

Microfiche copy: A01 
SAND94-0320

Distribution

May, 1994

Category UC-700

\title{
Analysis of Anelastic Flow and Numerical Treatment via Finite Elements
}

\author{
M. J. Martinez \\ Engineering Sciences Center \\ Sandia National Laboratories \\ Albuquerque, NM 87185
}

\begin{abstract}
In this report, we reconsider the various approximations made to the full equations of motion and energy transport for treating low-speed flows with significant temperature induced property variations. This entails assessment of the development of the so-called anelastic equations for low-Mach number flows outside the range of validity of the Boussinesq equations. An integral part of this assessment is the development of a finite elementbased numerical scheme for obtaining approximate numerical solutions to this class of problems. Several formulations were attempted and are compared.
\end{abstract}




\section{Acknowledgment}

The author thanks D. K. Gartling, J. N. Shadid and H. K. Moffat for many helpful discussions. This work was supported by the U. S. Department of Energy under contract DEAC04-76DP00789. 


\section{Table of Contents}

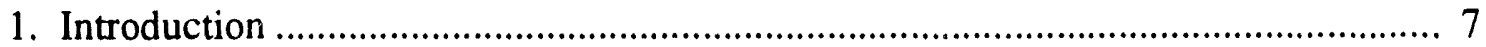

2. The Boussinesq and Anelastic Approximations....................................................... 8

2.1 The Boussinesq Approximation...................................................................... 9

2.2 The (Classical) Anelastic Approximation ...................................................... 10

2.3 Acoustically Filtered Equations ......................................................................... 11

2.4 Some Implications of the Anelastic Approximation.......................................... 13

2.5 Semi-Implicit Numerical Methods.................................................................. 14

3. Numerical Analysis ....................................................................................... 15

3.1 Spatial Discretization via Finite Element Approximations.................................. 16

3.2 Time Integration via Finite Difference........................................................ 18

3.2.1 Anelastic approximation.......................................................................... 18

3.2.2 Acoustically filtered approximation - density formulation $(\operatorname{AF}(\rho))$............. 19

3.2.3 Acoustically filtered appoximation - temperature formulation $(\mathrm{AF}(\mathrm{T}))$....... 19

3.3 Methods and Strategies Employed ................................................................ 20

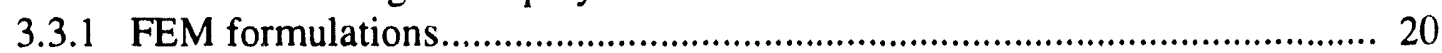

3.3.2 Time integration and solver strategies ..................................................... 20

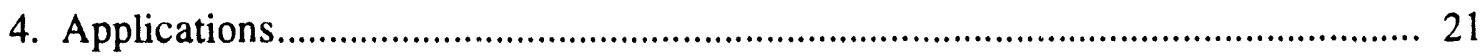

4.1 Steady Convection in a Box...................................................................... 21

4.1.1 Problem Description .............................................................................. 21

4.1.2 Discussion of Results.......................................................................... 21

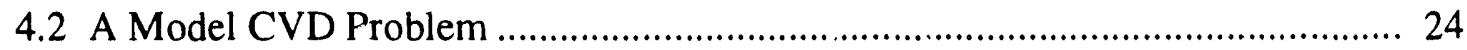

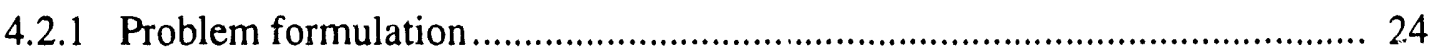

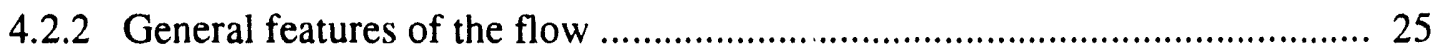

4.2.3 Gas expansion and the transient evolution ............................................ 30

4.2.4 Heat transfer and gas expansion ............................................................. 31

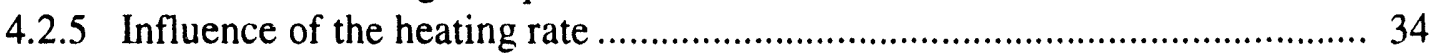

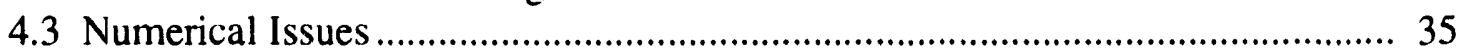

4.3.1 Performance of the methods .................................................................. 35

4.3.2 The AF formulations............................................................................. 36

4.3.3 Mesh-dependent pressure fluctuations …………….................................... 36

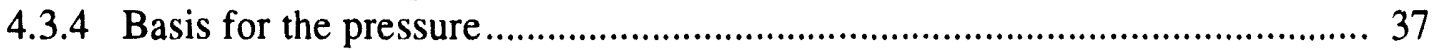

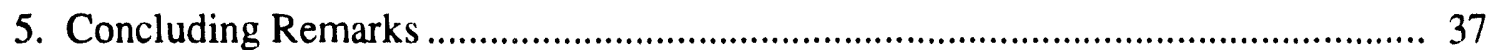

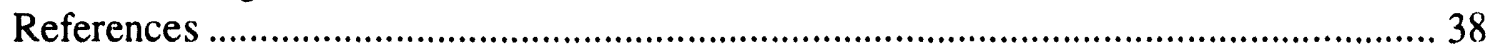

Newton Iteration ....................................................................................... 40 


\section{List of Figures}

Figure 1 Temperature contours for natural convection in a cavity due to a horizontal temperature difference.

Figure 2 Schematic of the model CVD problem.................................................. 24

Figure 3 Susceptor temperature heat-up history............................................... 26

Figure 4 The evolution of temperature for the anelastic formulation. ..................... 27

Figure 5 The evolution of the stream function for the anelastic formulation. ........... 28

Figure 6 Steady distribution of temperature and stream function for the Boussinesq formulation.

Figure 7 Steady distribution of temperature and stream function for the anelastic formulation..................................................................................... 29

Figure 8 Volume efflux history for the three different equation formulations. .......... 31

Figure 9 Mass efflux history for the three different equation formulations.............. 32

Figure 10 Error in the relation between heat transfer and the induced dilatation....... 32

Figure 11 Nusselt number history for the three different equation formulations......... 33

Figure 12 The influence of the heating rate on the global mass efflux from the computational domain........................................................................... 35 


\section{Introduction}

This work was motivated by the need for development of finite element schemes for treating problems with large temperature differences, for which the Boussinesq equations are of questionable validity. The treatment of non-Boussinesq flows via finite elements does not appear to have received as much attention as with finite difference methods (see Oran and Boris, 1987, for example). Therefore, the focus of this work was twofold: 1) to assess approximations that have been proposed for treatment of low-Mach number flows with large temperature differences, and 2) to develop and implement a finite element numerical scheme for treating this type of system. For the purpose of this discussion, low-speed flows are defined roughly as flows for which $M a<0.3$, which includes the incompressible and subsonic regimes. The main condition is that the flow speed is everywhere substantially less than the sound speed, and that variations in density are due mainly to variations in temperature and not pressure. It is expedient to simplify the full compressible equations of motion and energy for analyzing problems in this regime and this report discusses some of the approaches which have been considered and how they relate to the development of numerical methods.

In discussing the approximations, we consider both analytical and numerical approaches. Analytical methods include the Boussinesq approximation, the "anelastic equations" developed by geophysical fluid dynamicists and the related acoustically filtered equations. The latter system is also referred to as an anelastic approximation (Sherman, 1990), but for purposes of distinction, will be referred to as "acoustically filtered equations." Much of the early motivation for these approximations was to avoid time step limitations in obtaining approximate numerical solutions to problems in atmospheric studies. The time step limitations are imposed by the Courant-Friedrichs-Lewy (CFL) condition for explicit methods, which in this flow regime is based on the sound speed. Even though the CFL condition can be avoided by a fully implicit method, the Mach number will still determine the multiple of the CFL time step, $\Delta t_{C F L}$ say, that is required for accurate resolution. Even though an implicit method has no time step limitations for stability, too large a time step will yield an inaccurate solution. The system of equations becomes more hyperbolic in character as the Mach number increases, and therefore the time step required for accuracy will approach $\Delta t_{C F L}$ as $M a \rightarrow 1$. This is the reason high-speed flows are often computed with explicit methods, since particle velocities and sound speed are of the same order of magnitude. Recently, researchers have recognized that the time step restrictions can be relaxed somewhat by devising numerical schemes which treat certain terms implicitly and the remaining ones explicitly. These ideas are also discussed in the following.

The next section attempts to clarify the relation among the various approximations that have been invoked in simplifying the equations governing low-speed thermally driven flows (or at least flows for which thermal effects are significant). In addition, the role these analytical approximations play in development of numerical schemes will be noted, and various numerical schemes developed to treat low-speed, variable density flows are reviewed. In the following section a finite element method for obtaining numerical solutions to the anelastic equations is presented. The incompressible finite element code NACHOS II (Gartling, 1987) was used as a test bed for implementation of the anelastic equations. 
The numerical implementations of the various approximate methods are verified in the next section. The implementations are demonstrated on two example problems, steady natural convection in a box, and a model chemical vapor deposition (CVD) problem in a horizontal reactor configuration. Some numerical issues related to the performance of the implementations are also discussed.

\section{The Boussinesq and Anelastic Approximations}

The complete equations of motion for thermally driven flows are the continuity, momentum and energy equations:

$$
\begin{gathered}
\frac{D \rho}{D t}+\rho \nabla \bullet \boldsymbol{u}=0 \\
\rho \frac{D \boldsymbol{u}}{D t}=-\nabla p+\rho f+\nabla \bullet \boldsymbol{T} \\
\rho C_{p} \frac{D T}{D t}-\beta T \frac{D p}{D t}=\Phi-\nabla \bullet q
\end{gathered}
$$

where $\rho$ is density, $\boldsymbol{u}$ is the velocity vector, $p$ is pressure, $f$ is a body force vector,

$$
\mathbf{T}=2 \mu d-\frac{2}{3} \mu(\nabla \bullet u) I
$$

is the stress tensor,

$$
\boldsymbol{d}=\frac{1}{2}\left(\nabla \boldsymbol{u}+(\nabla \boldsymbol{u})^{T}\right)
$$

is the rate of deformation, $\beta$ is the thermal expansion coefficient, $\Phi=\mathrm{T}_{i j} d_{i j}$ is the dissipation, and $q=-k \nabla T$ is the heat flux vector. An equation of state is also needed

$$
\rho=\rho(T, p) \text {. }
$$

Embodied in this system is the full range of coupled phenomena involving fluid dynamics with energy transport. For many applications, it is desirable to eliminate some features which can a priori be shown to be unimportant to the problem at hand. In particular, for low Mach number flows, acoustic effects, pressure work and dissipation are normally unimportant and/or negligible. For this class of problems it is expedient to approximate the equations to reflect this situation. A formal way of arriving at the approximate equations is through a limiting process, i.e. an asympto ic expansion about a small parameter describing a ratio of physical processes. The following anelastic approximations were derived in this way. We will not reiterate these developments but only present the final approximate equations. (References to the original works are given.)

There are two so-called anelastic approximations, the original anelastic approximation (AN) developed for atmospheric flows and the more recently developed acoustically fil- 
tered approximation (AF). The latter is also an anelastic approximation in as much as elastic effects are eliminated by the approximation. The Boussinesq approximation will be indicated by $\mathrm{BO}$.

\subsection{The Boussinesq Approximation}

The earliest approximation made to the equations of motion for treating buoyantly driven flows was the so-called Boussinesq approximation. The basis for the approximation is that for some flows, temperature and density variations are small and yet the flow is driven by buoyancy. The Boussinesq approximation is useful to study flows which are firimarily the result of the baroclinic term, $(\nabla p \times \nabla \rho) / \rho^{2}$, in the vorticity equation. Spiegel and Veronis (1960) were apparently the first to interpret the Boussinesq approximation as the leading order system of equations in an expansion for small deviations in density from a basic state. The principal assumption is that $\Delta \rho / \rho \ll 1$. This assumption also restricts the scale height for motions in a gravity field $(f=g)$ such that if $d$ is the characteristic dimension for the motion, hydrostatic density variations must remain small over this scale. Writing $\rho=\rho_{0}+\rho "$, where $\rho_{0}$ is constant and $\rho "$ " $\rho_{0}$, and if characteristic values of velocity and time are measured by $U$ and $\tau$, respectively, the continuity equation, to lowest order in $\varepsilon=\Delta \rho / \rho_{0}$ becomes

$$
\nabla \bullet \tilde{u}=O(\varepsilon)
$$

so long as $d / U \tau \ll \varepsilon^{-1}$, where "tilded" variables are dimensionless. We note that this asymptotic limit does not claim constant density but only that $D \rho / D t=0$. The momentum equations are simplified by subtracting off the hydrostatic contributions, i.e., a motionless background state is assumed to exist, and we are interested in arriving at the momentum equations which describe the fluctuations about this basic state. For this purpose, variables are expanded according to

$$
p=p_{0}+p_{h}(z)+p^{\prime}(x, t)
$$

with similar expressions for density (where $\rho^{\prime \prime}=\rho_{h}(z)+\rho^{\prime}(x, t)$ ) and temperature. In these expansions, the subscript $h$ refers to the hydrostatic term and the primed quantity is the deviation we wish to solve for. Under these assumptions, the ideal gas equation of state can be used to show that, to leading order $\rho^{\prime}=-\beta T$, where $\beta=-(\partial \rho / \partial T)_{p} / \rho$. Implied in this equation is that the dynamic pressure fluctuations cannot compete with temperature fluctuations in modifying density. Using this approximation, and taking the buoyancy term as the ranking term in the dimensionless momentum equations results in the Boussinesq equations for low-speed flow:

$$
\begin{gathered}
\nabla \bullet \boldsymbol{u}=0 \\
\frac{D \boldsymbol{u}}{D t}=-\frac{1}{\rho_{0}} \nabla p^{\prime}-\beta T^{\prime} \boldsymbol{g}+\frac{\nabla \bullet(2 \mu d)}{\rho_{0}} \\
\frac{D T}{D t}=\kappa \nabla^{2} T
\end{gathered}
$$


where $T=T-T_{0}-T_{h}(z), \kappa=k /\left(\rho_{0} C_{p}\right)$, and the body force is taken to be gravity. In arriving at the low-speed energy equation, the pressure heating term $(\beta T(D p / D T))$ can be neglected if $d$ " $C_{p} \Delta T / \beta g T_{0}$. The dissipation can also be neglected if $d$ " $C_{p} / \beta g$, i.e., if the scale height for the motion is much smaller than the scale height for the adiabatic lapse rate, the temperature gradient in an isentropic atmosphere in hydrostatic equilibrium. By applying curl to the momentum equation, the vorticity equation in the Boussinesq approximation is

$$
\frac{D \omega}{D t}=(\omega \bullet \nabla) \boldsymbol{u}-\beta(\nabla T \times g)+\frac{1}{\rho_{0}} \nabla \times(\nabla \bullet(2 \mu d))
$$

where $\omega=\nabla \times u$. The equation shows that vorticity is generated by temperature-induced baroclinicity (in addition to vortex stretching) and implies that dynamic pressure fluctuations are incapable of generating vorticity. That is, in a gravity field, baroclinic generation is the result of horizontal temperature-induced density variations and the hydrostatic vertical pressure gradient.

Operationally, the outcome is that the density is treated as constant everywhere except in the body force driving the momentum equations. To summarize, the Boussinesq equations are restricted to small fluctuations in density and pressure relative to a static state, and to small scale height relative to the length scale over which fluid density is changed by adiabatic compression in a gravity field (this restriction is relevant to large scale atmospheric flows). The restriction to small motion-induced fluctuations in density and pressure is effectively a restriction to low Mach number. The restriction to small scale heights is relaxed by the anelastic approximation, to be discussed next.

\subsection{The (Classical) Anelastic Approximation}

The Boussinesq approximation, while having proven to be extremely useful, is nonetheless limited to $\Delta \rho / \rho$ \& 1 , a rather severe restriction. Ogura and Phillips (1962) derived the anelastic equations in the absence of molecular transport effects, and subsequently Gough (1969) extended the derivation to include these effects. The term "anelastic" was apparently coined by J. G. Charney (according to Ogura and Phillips, 1962) and refers to fluid motions in which elastic energy is absent, as in their approximate equations. The scale analysis of Ogura and Phillips is based on the assumptions of small temperature variation about an adiabatic state and a characteristic time scale associated with gravity waves. The time scale is given by the Brunt-Vaisala frequency, a frequency scale separating high frequency acoustic waves from lower frequency gravity waves. The analysis of Ogura and Phillips considered motions limited vertically by parallel fixed boundaries, and it is this restriction which principally leads to the requirement that $\partial \rho / \partial t$ vanish in the approximate continuity equation, perhaps the most notable result of this approximation. The approach taken by Gough (1969) is also to expand the equations under the condition that motion-induced density and temperature fluctuations are small. Gough works with the full compressible equations including conductive and viscous effects. He asserts that acoustic modes are eliminated as a result of $\partial \rho / \partial t=0$ in the continuity equation. This arises because of his assumption about the time scale for the motion, which is defined by the time required to travel the characteristic distance, $d$, the depth of his atmosphere, according to the characteristic vertical 
velocity, $w$. The latter is determined by the buoyancy-induced velocity, $w^{2}-g d \Delta T / T$. This leads to the result that temporal variations in density are asymptotically smaller than the divergence of mass flux.

The main advantage of the anelastic equations is that they relax the restriction to small scale heights, and also that the local time rate of change of density is eliminated. The resulting equations are still limited to small density fluctuations. It is of interest to note that Jarvis and McKenzie (1980) also describe an anelastic-liquid approximation as $\partial \rho / \partial t=0$, which is also claimed to eliminate acoustic modes in liquids, whereas the anelastic equations were derived for gases.

The anelastic equations are given by the following system,

$$
\begin{gathered}
\nabla \bullet \rho \boldsymbol{u}=0 \\
\rho \frac{D \boldsymbol{u}}{D t}=-\nabla p^{\prime}+\rho f+\nabla \bullet \boldsymbol{T} \\
\rho C_{p} \frac{D T}{D t}=-\nabla \bullet q \\
\rho=\rho(T),
\end{gathered}
$$

which can be compared with the complete system, eqns. (1-4). The equations presented above are really an (ad hoc) enhanced version of the equations presented by Gough (1969). In the original development of Gough, the density appearing in the balance equations is a horizontally averaged quantity. These equations were developed for atmospheric studies for which vertical scales are large enough to consider hydrostatic variations in density. In the present version, the continuity equation retains unrestricted variation, but only as a function of temperature, as stated by the approximated equation of state. The density is independent of dynamic pressure. Also important is that the local time rate of change of density is assumed negligible compared to the spatial divergence of mass. The pressure appearing in the momentum equation, $p^{\prime}$, is a second-order term in the asymptotic expansion (see Paolucci, 1982), and therefore represents an order $M a^{2}$ correction to the background pressure. The energy equation includes only heat conduction as contributing to the energy supply to fluid particles.

\subsection{Acoustically Filtered Equations}

Motivated by applications in fire research, Rehm and Baum (1978) were apparently the first to formally consider approximations to the equations of motion in the low Mach number regime which allow large density variations, i.e., they derived the acoustically filtered equations, albeit by neglecting dissipative effects. Rehm and Baum (1978) point out that acoustic modes are removed by uncoupling density and temperature fluctuations arising in the momentum equations from the equation of state. Therefore, the lowest order system of equations retains the continuity equation in its original form. This is in contrast to the assertion by Gough (1969) that acoustic modes are removed by replacing the full continuity equation with $\nabla \bullet \bar{\rho} \boldsymbol{u}=0$, where $\bar{\rho}$ is a horizontally averaged density (depends only on the 
elevation). Under the additional assumption that density variations are small, the acoustically filtered equations given by Rehm and Baum reduce to the Boussinesq equations (in the absence of molecular transport). The equations derived by Rehm and Baum (1978) were used in numerical simulations, using finite difference methods, of fire related convective transport problems (Baum et al., 1983, Baum and Rehm, 1984).

Le Quere et al. (1981) extended the equations of Rehm and Baum to include viscous and energy diffusion in a finite difference scheme applied to a solar receiver problem with large temperature differences. No formal derivation was given of the acoustically filtered equations. They computed solutions for Grashof numbers as high as $10^{7}$. By expanding the full compressible Navier-Stokes equations for small Mach number, Paolucci (1982) obtained a set of equations which do not include acoustic waves, but retain full density and material property variations. This development parallels that of Rehm and Baum (1978), but using the full equations including molecular transport effects (i.e. viscous and conductive mechanisms). Paolucci showed that his equations do not admit acoustic effects by developing the wave equation obeyed by the pressure. The resulting equation, in the limit $M a \rightarrow 0$, is elliptic, thereby showing that acoustic waves travel at infinite speed in this limit. The resulting equation, with further linearizing assumptions, is shown to be the linear equation governing internal waves. Paolucci (1990) and Chenoweth and Paolucci (1986) performed a series of numerical simulations based on these "enhanced" anelastic equations.

The basic outcome of the acoustically filtered equations is that the pressure is composed of a spatially independent background part plus an asymptotically smaller dynamical part,

$$
p=p_{0}(t)+M a^{2} p^{(1)}(x, t)+o\left(M a^{2}\right)
$$

where $p^{(1)}$ replaces,$p$ in the momentum equations, (2). The acoustic filtering comes about because the equation of state is written as

$$
\rho=\rho\left(T, p_{0}\right)
$$

Hence, the dynamical fluctuations in pressure do not enter into the equation of state. The remainder of the set of acoustically filtered equations includes the full continuity equation,

$$
\frac{\partial \rho}{\partial t}+\nabla \bullet \rho u=0
$$

while the momentum equation remains unchanged relative to the anelastic equations (eqn. (12), with $p^{\prime}=p^{(1)}$ from the expansion (15)), and the energy equation becomes

$$
\rho C_{p} \frac{D T}{D t}-\beta T \frac{d p_{o}}{d t}=-\nabla \bullet q
$$

In this approximation, the pressure $p_{0}$ represents the first order correction to the background pressure, and is governed by an energy balance of the flow system under consideration. If the system is open to the ambient, or when the flow is steady for example, $p_{0}$ is determined by the initial conditions of the system. Otherwise, if the fluid is held in a rigid container, 
then an energy balance on the system yields an evolution equation of the form (Paolucci, 1982)

$$
\frac{d p_{0}}{d t}=|\Omega|^{-1} \int_{\Gamma} q_{n} d \Gamma
$$

where $q_{n}$ is an applied heat flux.

In both the AF and AN approximations the pressure appearing in the momentum equation is decoupled from the equation of state. The idea is that the process is essentially isobaric and that the density of a fluid parcel varies due to isobaric thermal expansion. The dynamical pressure gradient is required to balance the momentum equation and satisfy continuity, but is too small to influence variations in density.

As regards numerical schemes, the acoustically filtered equations are not too different in form compared to the original equations, so that this approximation can be applied to an existing (compressible) algorithm with little modification. The major change would involve the addition of an evolution equation for $p_{0}(t)$, if necessary. In steady problems the only modification required is to decouple the dynamical pressure from the equation of state.

\subsection{Some Implications of the Anelastic Approximation}

The simplified equation of state, eqn. (16), shows that to this order of approximation,

$$
\frac{D \rho}{D t}=-\rho \beta \frac{D T}{D t}
$$

By combining this with the continuity and the energy equations (Sherman, 1990),

$$
\nabla \bullet \boldsymbol{u}=-\frac{\beta}{\rho c_{p}} \nabla \bullet q .
$$

The equation implies that dilatation is due solely to the addition of heat in the anelastic approximation to the full equations. Conversely, energy addition is a source of dilatation; consider the form for a calorically perfect gas,

$$
\int_{\Gamma} u \bullet n d \Gamma=-\int_{\Gamma} \frac{\beta}{\rho c_{p}} q \bullet n d \Gamma,
$$

indicating that any addition of heat into the domain $\Omega$ with boundary $\Gamma$, will result in an instantaneous net divergence of volume flux through the boundary. For a given heat input, this equation quantifies the extra volume flux (or velocity) to be expelled from the region. An interesting consequence, which will arise later in the applications, is that time-ramped temperature varying boundary conditions generate div $(\boldsymbol{u})$ at boundaries in proportion to the rate of heating specified. The case of an abruptly heated wall results in unbounded $\operatorname{div}(\rho \boldsymbol{u})$ and as such must represent an ill-posed problem for the AF system of equations. 
In a real gas, for example, such a boundary condition would result in acoustically generated motion of the surrounding fluid (see Spradley and Churchill 1975, for example).

Finally, we note that the pressure in anelastic flow, since it does not appear in the EOS, plays a similar role as in incompressible flow. The pressure will always be of the same order as the ranking term in the momentum equation (otherwise, the system of equations is over-determined without the pressure as one of the dependent variables). This also means that, in analogy to incompressible flow, the pressure plays the role of a Lagrange multiplier, required to satisfy continuity.

\subsection{Semi-Implicit Numerical Methods}

The foregoing reviewed the approximations made to the full equations of motion for treating the low-speed case. While these approximations are clearly important for understanding this flow regime and for devising efficient numerical schemes, their drawback is that the resulting equations are restricted at the outset by the assumptions and approximations made to derive them. Recently, researchers developing numerical methods for this flow regime have devised methods which, in a loose sense, relegate the acoustic filtering to the numerical scheme. The advantage is that codes can be written for the full equations of motion which include numerical schemes capable of treating a wider range of the parameter space (see chapter 9 of Oran and Boris, 1987). For example, the code could include an algorithm suitable for the acoustically filtered limit (semi-implicit formulations) and also an algorithm to treat higher speed flows (e.g. explicit methods, such as FCT).

Casulli and Greenspan (1984) discuss a pressure method for low-speed flows. The basic idea is that only certain terms in the equations need be treated implicitly, while the remaining formulation is explicit. By considering the characteristic equation for the inviscid, nonconducting compressible equations, they determine the terms from which arise the conditions on sound speed and therefore those terms which ought to be handled explicitly. These turn out to be the pressure terms in the Euler equations and the dilatational work in the energy equation. Ultimately, assuming an ideal, calorically perfect gas $(p=(\gamma-1) \rho e)$, the energy equation is written as an equation for the pressure. This version of the energy equation forms the basis of their iteration scheme. The iteration scheme is outlined in the paper and consists of a method to solve for the pressure and velocity simultaneously. Casulli and Greenspan demonstrate their method on a model vortex street problem using the full Navier-Stokes equations, but neglecting molecular transport in the energy equation. They were able to use a time step which was about ten times the step allowed by the CFL condition based on the acoustic speed.

Motivated by the work of Casulli and Greenspan, Patnaik et al. (1986) developed a "barely implicit correction" (BIC) to a flux-corrected transport (FCT) algorithm suitable for treating subsonic flows. They introduce an implicitness parameter, similar to the "theta" parameter used in time integration, into their formulation which can be used to advantage when sound or pressure waves are important in subsonic flows. The idea of the BIC is to formulate a semi-implicit algorithm which adds an elliptic equation for a pressure correction to be solved together with explicit equations for updating velocity and energy. This method 
relaxes the time step restriction which would otherwise have to be observed by an explicit algorithm, in this case FCT.

Karki and Patankar (1988) consider a pressure-based finite difference method for application to "all speeds." Pressure is chosen as a dependent variable since, for low Mach number, coupling between density and pressure is weak, hence a density-based method is likely to fail. They note that whereas density may be only mildly variable at low-speed, pressure will always have finite variation at all speeds. They also note that most incompressible algorithms are based on solving for pressure, whereas compressible (high Mach number) methods normally solve for density and obtain pressure from an equation of state. Their algorithm utilizes a compressible form of the SIMPLER method.

The method discussed by Mueller (1992) may also be loosely categorized under the semiimplicit techniques discussed here. Mueller developed a conjugate graciient method for low Mach number flows based on the idea of an embedded solution for pressure. He considers only isothermal problems, but it appears his method could be extended to include an energy equation. The formulation of his equations is also efficient for finite element methods, although his scheme is not tied to any particular discretization method. Computational results from his algorithm look promising.

Also under the title of semi-implicit methods, we mention the vortex-based method discussed by Majda and Sethian (1985). These authors consider combustion of premixed fuel under simplified assumptions for the chemical reaction; they consider a two species system, burnt and unburned gas. The unburned gas is converted to burnt gas via a one-step irreversible Arrhenius kinetics mechanism. Assuming ideal gas, they work with a "pressure equation" in place of continuity. The pressure equation is derived by combining continuity, energy, and the ideal gas equation of state. Even though Majda and Sethian arrive at the same conclusion as Rehm and Baum (1978) and Paolucci (1982) regarding the superposition of pressure as a spatially independent part and a dynamical fluctuation, they get there by a different route. Perhaps the biggest difference is that by using the decomposition $\boldsymbol{v}=\boldsymbol{w}+\nabla \varphi$, with $\nabla \bullet w=0$, where $\boldsymbol{v}$ is the desired velocity vector, an elliptic equation for $\varphi$ is generated. $\varphi$ then appears in an incompressible momentum equation. That is, they use projection on the original equations rather than on the discretized equations. They solve their incompressible momentum equation by the (two-dimensional) vortex method.

\section{Numerical Analysis}

In this section we outline the numerical treatment, using finite element methods, of the foregoing anelastic approximations. The formulations were implemented using NACHOS II (Gartling, 1987) as a platform. The treatment of the Boussinesq equations is discussed by Gartling. The present discussion is similar in many respects to that of Gartling, and that work can be consulted for further details about the application of the finite element method to incompressible flow. 


\subsection{Spatial Discretization via Finite Element Approximations}

Spatial discretization of the governing equations was accomplished by the Galerkin finite element method (GFEM, see Gartling, 1987). First, the velocity, temperature, pressure, and density fields are approximated by a finite-dimensional basis,

$$
u_{i}=\Phi^{T} u_{i}, \quad T=\Phi^{T} T, \quad p^{\prime}=\Psi^{T} P, \quad \rho=\Phi^{T} \rho .
$$

Substitution of the approximate fields in the acoustically filtered equations and application of the GFEM results in the discrete form of the continuity, momentum and energy equations,

$$
\begin{gathered}
H \dot{\rho}+Q_{i}^{T} R u_{i}=0 \\
M \dot{u}_{i}+C_{j}\left(R u_{j}\right) u_{i}-Q_{i} P+\tilde{K}_{i j} u_{j}+B_{i}=F_{i} \\
N \dot{T}+D_{i}\left(R u_{i}\right) T+L_{i i} T=G(T)
\end{gathered}
$$

respectively, where

$$
\begin{gathered}
\boldsymbol{H}=\int_{\Omega} \Psi \Phi^{T} d \Omega \\
\boldsymbol{Q}_{i}=\int_{\Omega} \frac{\partial \Phi}{\partial x_{i}} \Psi^{T} d \Omega \\
M=\int_{\Omega} \rho \Phi \Phi^{T} d \Omega \\
\boldsymbol{C}_{i}\left(\boldsymbol{R} \boldsymbol{u}_{i}\right)=\int_{\Omega} \Phi \Phi^{T} R u_{i} \frac{\partial \Phi^{T}}{\partial x_{i}} d \Omega \\
\boldsymbol{K}_{i j}=\int_{\Omega} \mu \frac{\partial \Phi}{\partial x_{i}} \frac{\partial \Phi}{\partial x_{j}} d \Omega \\
\tilde{\boldsymbol{K}}=\left[\begin{array}{l}
\frac{4}{3} K_{11}+K_{22} K_{21}-\frac{2}{3} K_{12} \\
K_{12}-\frac{2}{3} K_{21} K_{11}+\frac{4}{3} K_{22}
\end{array}\right]
\end{gathered}
$$




$$
\begin{gathered}
\boldsymbol{B}_{i}=\int_{\Omega} \Phi \Phi^{T} \rho g_{i} d \Omega \\
\boldsymbol{F}_{i}=\int_{\partial \Omega} \Phi \boldsymbol{T}_{i j} n_{j} d \Gamma \\
\boldsymbol{N}=\int_{\Omega} \rho c_{p} \Phi \Phi^{T} d \Omega \\
\boldsymbol{D}_{i}\left(\boldsymbol{R} \boldsymbol{u}_{i}\right)=\int_{\Omega} c_{p} \Phi \Phi^{T} \boldsymbol{R} u_{i} \frac{\partial \Phi^{T}}{\partial x_{i}} d \Omega \\
\boldsymbol{L}_{i i}=\int_{\Omega} k \frac{\partial \Phi}{\partial x_{i}} \frac{\partial \Phi^{T}}{\partial x_{i}} d \Omega \\
\boldsymbol{G}=\int_{\Omega} \Phi Q d \Omega+\int_{\partial \Omega} \Phi q_{i} n_{i} d \Gamma
\end{gathered}
$$

and $\boldsymbol{R}=\operatorname{diag}[\rho]$. This system is augmented in the non-Boussinesq formulation with an equation of state (EOS)

$$
\rho=\rho(T) .
$$

This formulation utilizes the mixed method of GFEM for incompressible flow wherein the basis for velocity and temperature, $\Phi$, is one order lower than that for pressure, $\Psi$. Here we will assume the anelastic equations must also satisfy the same criteria, i.e., the pressure appearing in the momentum equations is decoupled from the equation of state, and therefore plays the role of a Lagrange multiplier required to satisfy the continuity equation, a constraint on the system.

It may be of some interest to note the difference between this formulation and the corresponding incompressible formulation in NACHOS II. For the anelastic formulations, the variable density affects the local time derivatives, the convective accelerations, and the body force in the momentum equation. The variable density is included in the convective terms via use of the $\boldsymbol{R}$ matrix. By use of this matrix, the present formulation utilizes the same space-dependent FE matrices as are used in incompressible flow for the convective accelerations, viz., $\boldsymbol{C}$ and $\boldsymbol{D}$. The variable density involves the inner product of $\boldsymbol{R}$ with these matrices. This formulation thus allowed easy implementation of the anelastic approximations into NACHOS II. The other major difference is in how the body force term is handled. The present formulation implements the body force term directly, compared to the temperature-based formulation required by the Boussinesq approximation in an incompressible code. In the present version, the mass matrix $\boldsymbol{M}$ is approximated by an element-averaged density weighting. The only new matrices required $\operatorname{are} \boldsymbol{B}$ and $\boldsymbol{H}$. 


\subsection{Time Integration via Finite Difference}

To obtain recursion relations for time integration, consider the finite-difference approximation (Gresho, 1979, Gartling, 1987),

$$
\dot{V}_{n+1}=\frac{\eta}{\Delta t}\left(V_{n+1}-V_{n}\right)-(\eta-1) \dot{V}_{n}
$$

where the subscript indicates the time level, $\Delta t=t_{n+1}-t_{n}$, and $\eta=1$ for backward Euler and $\eta=2$ for the trapezoid rule. Note that this formula is different from the typical second-order Crank-Nicolson scheme (Huebner and Thornton, 1982). In the latter, the time-dependent equations are evaluated at the mid-time-plane, to obtain the second-order approximation. In the present formula, second-order in time is also achieved, but by a formula that involves the time-derivative at the desired timeplane, $t_{n+1}$. The second-order is achieved here by including time derivatives at the previous time-plane, e.g. $\dot{V}_{n}$. Substituting this approximation for the time derivatives in the spatially discretized system yields a nonlinear system of equations to be solved for the velocity, pressure and temperature at node points.

\subsubsection{Anelastic approximation}

Substituting (40) into the spatially discretized acoustically filtered equations, eqns. (2426 ), yields the following system

$$
\begin{gathered}
{\left[\begin{array}{cccc}
\frac{\eta}{\Delta t} M+C+\tilde{K}_{11} & \tilde{K}_{12} & -Q_{1} & 0 \\
\tilde{K}_{21} & \frac{\eta}{\Delta t} M+C+\tilde{K}_{22} & -Q_{2} & 0 \\
-Q_{1}^{T} R & -Q_{2}^{T} R & 0 & 0 \\
0 & 0 & 0 & \frac{\eta}{\Delta t} N+D+L
\end{array}\right]\left[\begin{array}{l}
u_{1} \\
u_{2} \\
P \\
T
\end{array}\right]=\left[\begin{array}{c}
F_{1}-B_{1} \\
F_{2}-B_{2} \\
-H \dot{\rho} \\
G
\end{array}\right]+} \\
\left(\frac{\eta}{\Delta t}\right)\left[\begin{array}{c}
M u_{1 n} \\
M u_{2 n} \\
0 \\
N T_{n}
\end{array}\right]+(\eta-1)\left[\begin{array}{c}
M \dot{u}_{1 n} \\
M \dot{u}_{2 n} \\
0 \\
N \dot{T}_{n}
\end{array}\right]
\end{gathered}
$$

where $C=C_{i}\left(R u_{i}\right), D=D_{i}\left(R u_{i}\right)$, and $L=L_{i i}$. Here and in the remainder of this section, variables evaluated at the current time level, $t_{n+1}$, are unsubscripted, whereas variables from the previous time level are indicated. The pressure is associated with the continuity equation, resulting in a zero on the diagonal for the associated submatrix of the full system of equations. This is not a problem for the NACHOS II solver which utilizes a direct (frontal) scheme. 
The anelastic equations are recovered by setting the RHS to zero in the continuity block. The acoustically filtered equations were formulated in two different ways, depending on how the $\rho$ term is treated.

\subsubsection{Acoustically filtered approximation - density formulation (AF( $\rho)$ )}

In the present scheme the density in the continuity equation is treated as a nonlinear temperature dependent property. This only modifies the continuity equation with respect to the system shown in (41), and only the continuity block (the third row in (41)) is shown below,

$$
\left[\left(-Q_{1}^{T} \boldsymbol{R}\right)\left(-Q_{2}^{T} \boldsymbol{R}\right) \mathbf{0} 0\right]\left[\begin{array}{l}
u_{1} \\
u_{2} \\
P \\
T
\end{array}\right]=\left[\frac{\eta}{\Delta t} \boldsymbol{H}\left(\rho-\rho_{n}\right)-(\eta-1) H \dot{\rho}_{n}\right]
$$

with the approximation

$$
\dot{\rho}_{n} \approx \frac{\partial \rho}{\partial T}\left(T_{n}\right) \dot{T}_{n}
$$

The latter approximation avoids the need to store $\dot{\rho}_{n}$, and allows its approximation using only primary variables, the values of which must be stored from the preceding time step for the two-step time integration scheme. Otherwise, this formulation is nothing more than an application of the recursion relation (40) to the RHS of the continuity block in (41). Note that the temperature dependence of density in the body force term and the continuity equation add to the nonlinearities in the force vector in (41). The resulting nonlinear system is solved via Newton's method, where the temperature dependence of density on the RHS of (42) is taken into consideration. The Newton iteration scheme is outlined in the appendix.

\subsubsection{Acoustically filtered appoximation - temperature formulation ( $A F(T))$}

A slightly different formulation was also implemented where the temperature dependence of density is incorporated into the time dependent ODEs before applying the recursion relation for time derivatives. Here we use $\dot{\rho}=(\partial \rho / \partial T) \dot{T}$ such that the continuity equation is

$$
-H \boldsymbol{R}^{\prime} \dot{T}-\boldsymbol{Q}_{i}^{T} \boldsymbol{R} \boldsymbol{u}_{i}=\mathbf{0}
$$

where

$$
\boldsymbol{R}^{\prime}=\operatorname{diag}\left[\frac{\partial \rho}{\partial T}\right]
$$

Applying the recursion relation yields the continuity block, 


$$
\left[\left(-Q_{1}^{T} R\right)\left(-Q_{2}^{T} R\right) 0\left(-\frac{\eta}{\Delta t} H R^{\prime}\right)\right]\left[\begin{array}{l}
u_{1} \\
u_{2} \\
P \\
T
\end{array}\right]=\left[-\frac{\eta}{\Delta t} H R^{\prime} T_{n}-(\eta-1) H R^{\prime} T_{n}\right]
$$

This formulation brings out the temperature dependence of $\dot{\rho}$ explicitly. The Newton iteration scheme for this nonlinear system is given in the appendix.

\subsection{Methods and Strategies Employed}

As noted in the introduction, an objective of this work was to demonstrate a finite element method for the anelastic equations. Therefore, several numerical methods and solver strategies were tested in an attempt to find a superior scheme. The several implementations are discussed in the 1ollowing.

\subsubsection{FEM formulations}

The incompressible finite element code NACHOS II was used as a platform for implementing the various anelastic approximations. The so-called mixed method was implemented in which the basis for velocity and temperature was the nine-node biquadratic approximation. The pressures were represented with either bilinear or discontinuous linear elements.

\subsubsection{Time integration and solver strategies}

Two time integration schemes are available in NACHOS II and were retained for the present work. A first-order-in-time scheme is operational which uses a forward Euler predictor and a backward Euler corrector. This scheme was only implemented with a Picard iteration scheme, and as such is not very efficient for the anelastic equations.

A second order method utilizes an Adams-Bashforth predictor with a trapezoid rule corrector. Both this and the previous time integrators include an adaptive time-stepping scheme, as discussed in Gartling (1987), which attempts to keep the local time truncation error at a user-specified level. This is accomplished by estimating the local time truncation error based on an error norm between the predictor and corrector solutions. The resulting nonlinear system is solved with a Newton iteration scheme, including (occasional) cycling of the corrector step. NACHOS II implements a one-step Newton method, whereby the corrector equation is not iterated. This is not to say the one-step Newton method is inaccurate. This strategy was advocated by Gresho et al. (1980) who noticed that the above-mentioned adaptive time-stepping scheme, with sufficiently small error tolerance required, on average, only one Newton iteration to achieve convergence at each time step. This strategy works well for the incompressible problem, but is not as secure a strategy for the anelastic equations, apparently because the latter are more nonlinear owing to the temperature variations of density, especially in the continuity equation. Thus, the current implementation includes an occasional iteration of the corrector to convergence, defined such that the re- 
sidual error is reduced to some fraction of the user-specified tolerance on the time integration, $\varepsilon_{\imath}$. A strategy to iterate the corrector requires at least two iterations, if only to test for convergence. The strategy that was ultimately implemented was devised to (hopefully) reduce the overall work by the integrator. The corrector is only iterated if the error norm exceeds a user-specified multiple of $\varepsilon_{t}$. Another viewpoint on the strategy is that the corrector is only iterated if, based on the current provisional solution vector, the time-step algorithm would reduce the subsequent step by more than a specified value (one-half, for example). Thus, the time integrator attempts to use the one-step Newton scheme until (or unless) the time truncation error is "too large", in which case the corrector is iterated to convergence.

A time-step restart scheme was also implemented. That is, if the time integration fails to converge after a specified number of iterations, the current time step is rejected, and a new time step is attempted with half the time step size.

\section{Applications}

The implementation of the various numerical formulations for anelastic (AN and AF) flow is demonstrated in this section. Two problems are considered, both involve convection with large temperature differences. Numerical results for the first problem are available for comparison. Boussinesq solutions were obtained with NACHOS II and can also serve as a basis for comparing general features of the ensuing flows.

\subsection{Steady Convection in a Box}

\subsubsection{Problem Description}

The implementation for steady flow was verified by comparison with published results (Chenoweth and Paolucci, 1986) for natural convection in a box. Because the flow is steady, there is no distinction between the AF and AN equations. The cavity temperature is specified as $T_{\mathrm{H}}$ on the left wall, $T_{C}$ on the right side, and no-heat-flux conditions top and bottom. The no-slip condition is specified for the velocity vector at all walls. The gas in the cavity is air for which the Sutherland laws for the temperature variation of viscosity and conductivity were used. The results were computed on a uniformly spaced $20 \times 20$ mesh of 9-node biquadratic elements for velocity and temperature. The pressure was represented by the discontinuous linear element (Gartling, 1987).

\subsubsection{Discussion of Results}

An example comparison is shown in Figure 1 between the Boussinesq solution for the temperature distribution, generated with NACHOS-Il, and the non-Boussinesq solution, both for a temperature difference $\varepsilon=\left(T_{H}-T_{C}\right) /\left(T_{H}+T_{C}\right)=0.6$, which is well outside the Boussinesq limit. The most notable difference is the asymmetry in the solution for the non-Boussinesq case. In contrast the Boussinesq equations are centrosymmetric as illustrated in the solution determined with NACHOS-II. Plots of the stream function (not shown) show that the primary vortex is centrally located for the Boussinesq solution, 


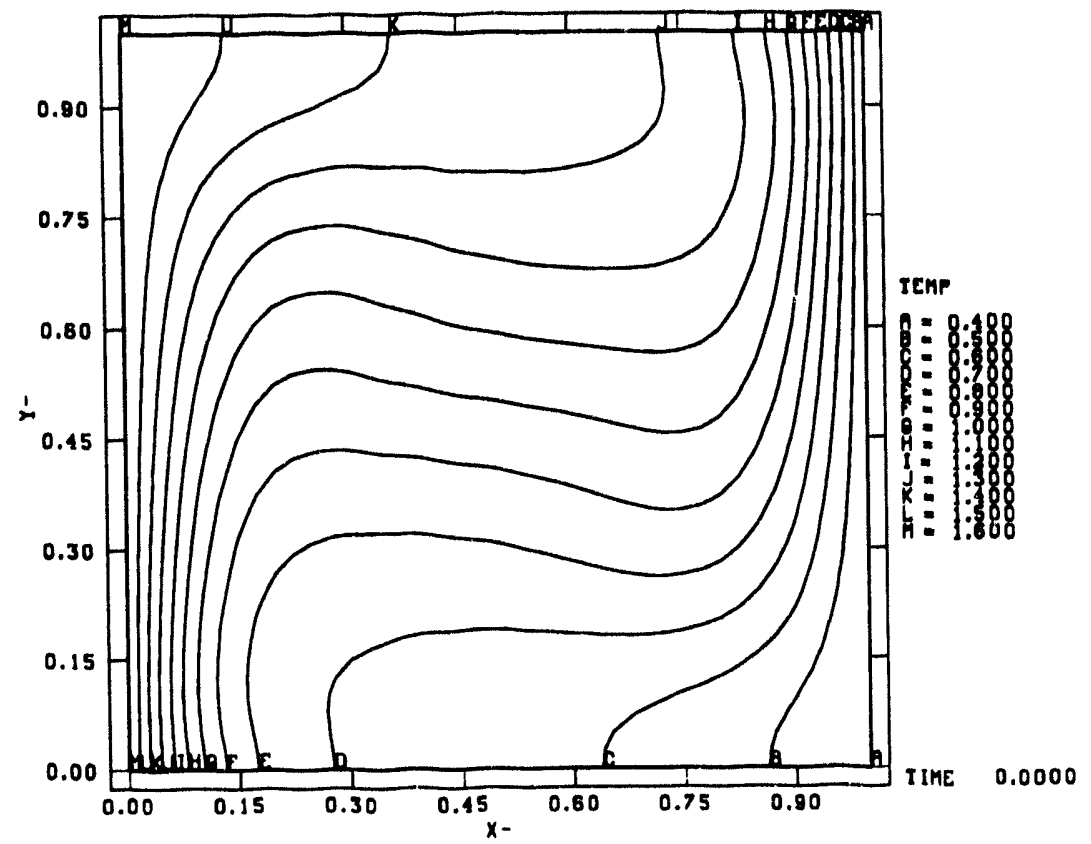

(a)

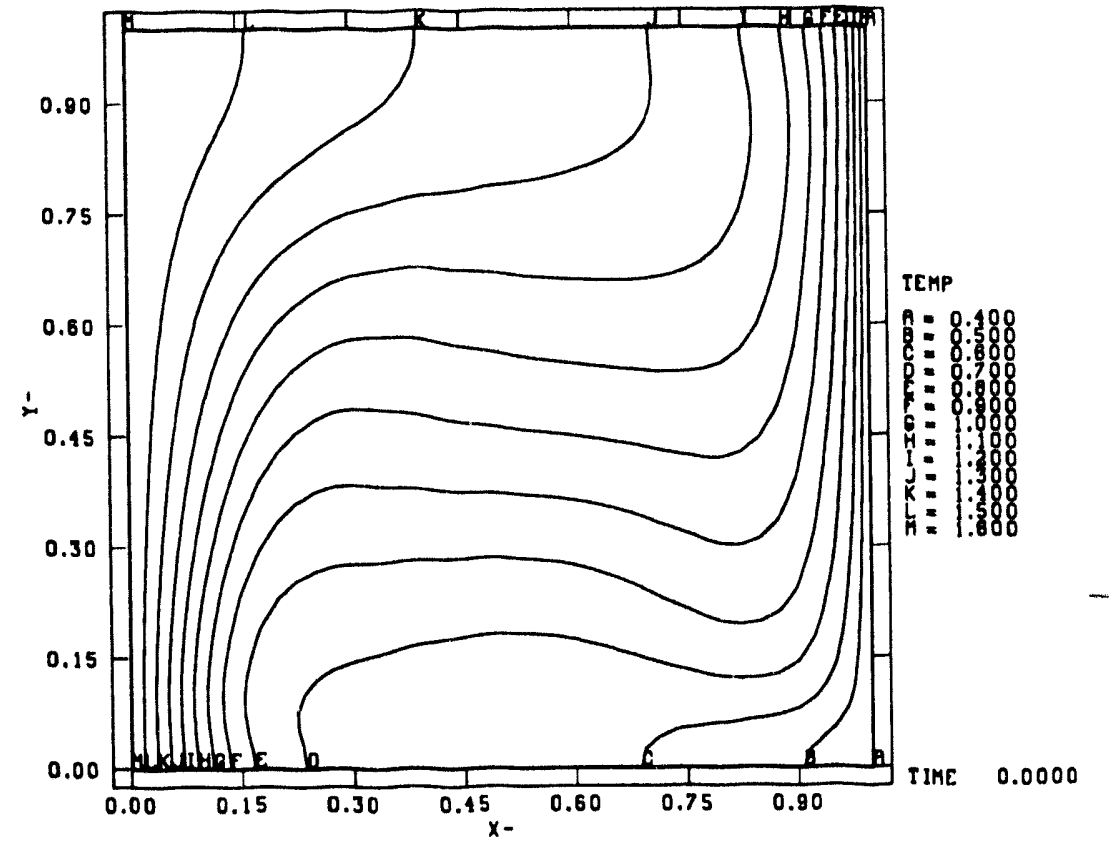

(b)

Figure 1 Temperature contours for natural convection in a cavity due to a horizontal temperature difference. The comparison is between the a) Boussinesq and b) anelastic solutions for $\mathrm{Ra}=50,000$ and $\varepsilon=0.6$. 
while the vortex is located towards the lower corner of the cold wall in the non-Boussinesq solution. This can be understood simply as an effect of variable properties. The net heat flux through the cold and hot walls must be equal. Since the conductivity of gases increases with temperature, the temperature gradient normal to the cold wall must, on average, exceed the gradient on the hot wall. The non-Boussinesq solution with constant properties will not require this asymmetry in temperature gradient to maintain uniform heat flow.

Table 1 compares results for the calculation of average Nusselt number ( $\mathrm{Nu})$, for the total heat flow across the cavity, as a function of Rayleigh number $(\mathrm{Ra})$. The results computed with the non-Boussinesq code compare well with the results given in Chenoweth and $\mathrm{Pa}$ olucci (1986), and with NACHOS-II in the Boussinesq limit $(\varepsilon=0.005)$. Presumably, the use of graded meshes, as used in Chenoweth and Paolucci (1986), would improve the agreement.

Surprisingly, the results for unit aspect ratio reported by Chenoweth and Paolucci (1986) for the average Nusselt numbers in the non-Boussinesq regime (up to $\varepsilon=0.6$ ) are the same as the corresponding Boussinesq values for constant properties. Given the differences between the Boussinesq and non-Boussinesq solutions as illustrated in Figure 1 it is not clear that this should be the case. Clearly, the temperature and velocity fields are different between the two approximations. Nusselt number values were computed which were in error by about $8 \%$ when specifying constant $\mu$ and $k$ in the non-Boussinesq formulation. However, the Boussinesq values are recovered when using the temperature-dependent properties as reported in Chenoweth and Paolucci (1986). Of course constant properties are consistent with the Boussinesq approximation, whereas constant properties in the nonBoussinesq problem represent a fictitious fluid rather than air. However, it is somewhat surprising that the Boussinesq equations happen to give the correct answer (for the average Nusselt number) to a problem for which the Boussinesq approximation is invalid.

Table 1: Comparison of Nusselt Numbers

\begin{tabular}{|l|l|l|l|}
\hline \multicolumn{1}{|c|}{$\mathrm{Ra}$} & \multicolumn{1}{c|}{$\varepsilon$} & \multicolumn{1}{c|}{$\mathrm{Nu}$} & \multicolumn{1}{c|}{ Reference } \\
\hline \hline $10^{4}$ & 0.005 & 2.244 & ${\mathrm{C} \& \mathrm{P}^{\mathrm{a}}}^{-1}$ \\
\hline $10^{4}$ & 0.005 & 2.253 & NACHOS-II \\
\hline $10^{4}$ & 0.005 & 2.261 & $\mathrm{AN}^{\mathrm{b}}$ \\
\hline $10^{4}$ & 0.6 & 2.244 & $\mathrm{C} \& \mathrm{P}$ \\
\hline $10^{4}$ & 0.6 & 2.293 & $\mathrm{AN}$ \\
\hline $5 \times 10^{4}$ & 0.6 & 3.66 & ${\mathrm{C} \& \mathrm{P}^{\mathrm{c}}}^{4}$ \\
\hline $5 \times 10^{4}$ & 0.6 & 3.70 & $\mathrm{AN}$ \\
\hline
\end{tabular}

a. Chenoweth and Paolucci, JFM. 169, pp.173-210, 1986.

b. Non-Boussinesq version of NACHOS-II

c. Interpolated from Table 2. 


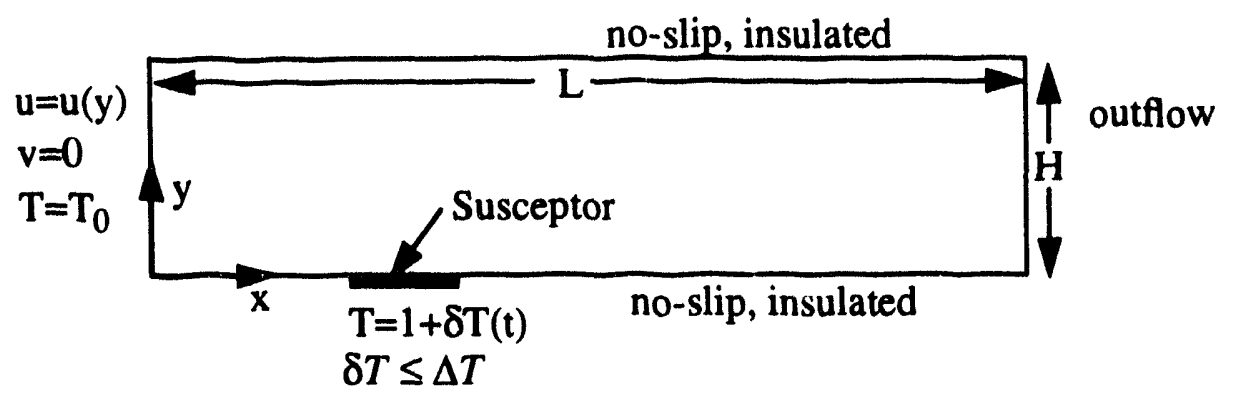

Figure 2 Schematic of the model CVD problem.

\subsection{A Model CVD Problem}

In this section we present results of simulations for a problem typical of those for which this numerical scheme was intended. We consider the start-up problem for flow of a gas through a channel heated from below, see Figure 2. This problem is similar to the socalled horizontal reactor in the chemical vapor deposition (CVD) process (Jensen, 1987).

\subsubsection{Problem formulation}

If the anelastic equations (including the AF equations) are nondimensionalized, the following parameters appear in the problem,

$$
R e=\frac{\rho_{0} U H}{\mu_{0}} \quad \operatorname{Pr}=\frac{v_{0}}{\alpha_{0}} \quad R i=\frac{G r}{R e^{2}}=\frac{g H \varepsilon}{U^{2}} \quad \varepsilon=\frac{\Delta T}{T_{0}}
$$

which are a Reynolds number, Prandtl number, Richardson number, and a temperature difference parameter, respectively. Also included in the set of parameters is the aspect ratio $L / H$, and the susceptor heating rate, $\dot{T}_{w}$ (to be discussed below). Here the velocity is scaled by $U$, the entering volume flux per unit area, length by $H$, time by $H / U$, temperature by $T_{0}$, and pressure by $\rho_{0} U^{2} . v$ is the kinematic viscosity and $\alpha$ is the thermal diffusivity. The subscript indicates temperature dependent properties evaluated at $T_{0}$. Variables in the remainder of this section are dimensionless.

A perfect-gas equation of state is also assumed,

$$
\rho T=1 .
$$

The initial condition is a two-dimensional Poiseuille flow distribution and uniform temperature, $T=T_{0}=1$. These conditions are maintained at the inlet plane throughout the remainder of the simulation. The upper and lower walls are no-slip (including the recently 
fashionable statement of no-penetration) boundaries and, except for the susceptor, are insulated to heat flow.

The outflow conditions require special consideration. The desired condition is one such that it approximates a very long channel and which causes a minimum of influence upstream of its application in the truncated domain. The usual conditions specified in finite difference work are that

$$
\frac{\partial T}{\partial x}=\frac{\partial u}{\partial x}=\frac{\partial v}{\partial x}=0
$$

see for example Evans and Greif (1990). Unfortunately, these conditions are incompatible with the current finite element formulation, for which a specification of the total traction is the natural boundary condition. However, if a steady state exits, far downstream of the susceptor, the fluid will have acquired a uniform temperature distribution. Further upstream will be a region for which the viscous stresses are small compared to buoyant forces; this condition is specified by large values of the $R i$ number. Under these conditions the traction is mostly due to the pressure, and the pressure will be hydrostatic (but with temperature dependent density), again because of large $R i$ number. Hence, the outflow condition was specified as

$$
\mathrm{T}_{n n}=\mathrm{T}_{x x}=-\frac{R i}{\varepsilon}(\rho-1) y
$$

with the vertical velocity component and streamwise temperature gradient set to zero. In the Boussinesq case, the condition is given by

$$
\mathrm{T}_{n n}=\mathrm{T}_{x x}=-\frac{R i}{\varepsilon}(T-1) y .
$$

For the present application, the dimensionless momentum equation was written in the form,

$$
\rho \frac{D u}{D t}=-\nabla p^{\prime}+\frac{1}{R e} \nabla \cdot T+\frac{R i}{\varepsilon}(\rho-1) \nabla y
$$

so the pressure is the departure from the background hydrostatic value $\left(\rho_{0} g y\right)$.

Convection is initiated with the heat-up of the susceptor. The time history of the heat-up, shown in Figure 3, involves a linear temperature rise until the final operating temperature, $T_{H}$, is attained.

\subsubsection{General features of the flow}

To demonstrate the evolution of convection in the model CVD reactor, we choose the parameters: $R e=10, P r=1, R i=500\left(\mathrm{Gr}=5 \times 10^{4}\right), \varepsilon=0.5$ and $L / H=5$. As we shall see, this combination of Reynolds and Richardson numbers results in a significant buoyancydriven force active in the early evolution. The susceptor is $0.5 \mathrm{H}$ in length and begins $0.5 \mathrm{H}$ 


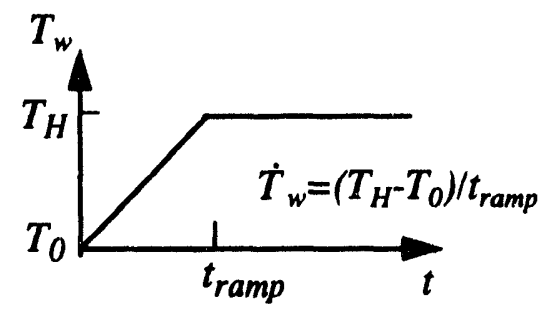

Figure 3 Susceptor temperature heat-up history.

units of length past the inlet plane. The time-ramp was applied with $t_{\text {ramp }}=0.05$, $T_{H}=1.5$, resulting in $\dot{T}_{w}=10$ during the susceptor heat-up phase.

The problem was solved using each of the three formulations, Boussinesq (BO), anelastic (AN) and acoustically filtered (AF). Constant thermal conductivity and viscosity were specified for the BO simulations, since this is consistent with this set of equations. For purposes of comparison, the anelastic equations were solved both for constant and temperature-dependent dissipative coefficients. The Sutherland laws were applied for the temperature-dependence, using values for air as given in White (1974). It is noted that the value $\varepsilon=0.5$ is outside the region of validity of the Boussinesq approximation, however, the BO simulation is performed for the sake of comparison with the anelastic solutions.

The simulation results to follow were obtained on a $55 \times 15$ element mesh. The nine-node biquadratic element was specified for representing velocity and temperature, and the fournode bilinear element for pressure. The mesh spacing was graded toward the region of the susceptor. Results due to mesh variation are discussed in the next section.

The evolution of convection is illustrated in Figures 4 and 5 which show the development of the temperature and stream function during the early transient initiated by the rapid heating of the susceptor. This solution was generated with the anelastic formulation. The evolution is similar for the BO and AF solutions, however, the stream function is only defined for the latter formulation at steady state. For this combination of $R e$ and $R i$ numbers, a fairly strong thermal develops above the plate. The $R i$ number is large enough to allow the thermal to rise to the top of the channel before being swept back by the incoming flow and eventually out the domain.

Reverse flow is initiated at the downstream end of the susceptor, owing to the thermal, leading to the development of a buoyant plume and a clockwise vortex behind the susceptor. An anti-clockwise vortex is also generated towards the top of the channel. The clockwise cell elongates towards the exit and eventually disappears, as shown in the steady solutions, Figures 6 and 7.

The pressure computed in these simulations is the departure from hydrostatic due to the dynamics of the evolution. This disturbance pressure develops a vertical gradient above the plate and eventually attains a nearly hydrostatic distribution behind the plate, i.e., the pressure varies with elevation but not in the streamwise direction. 

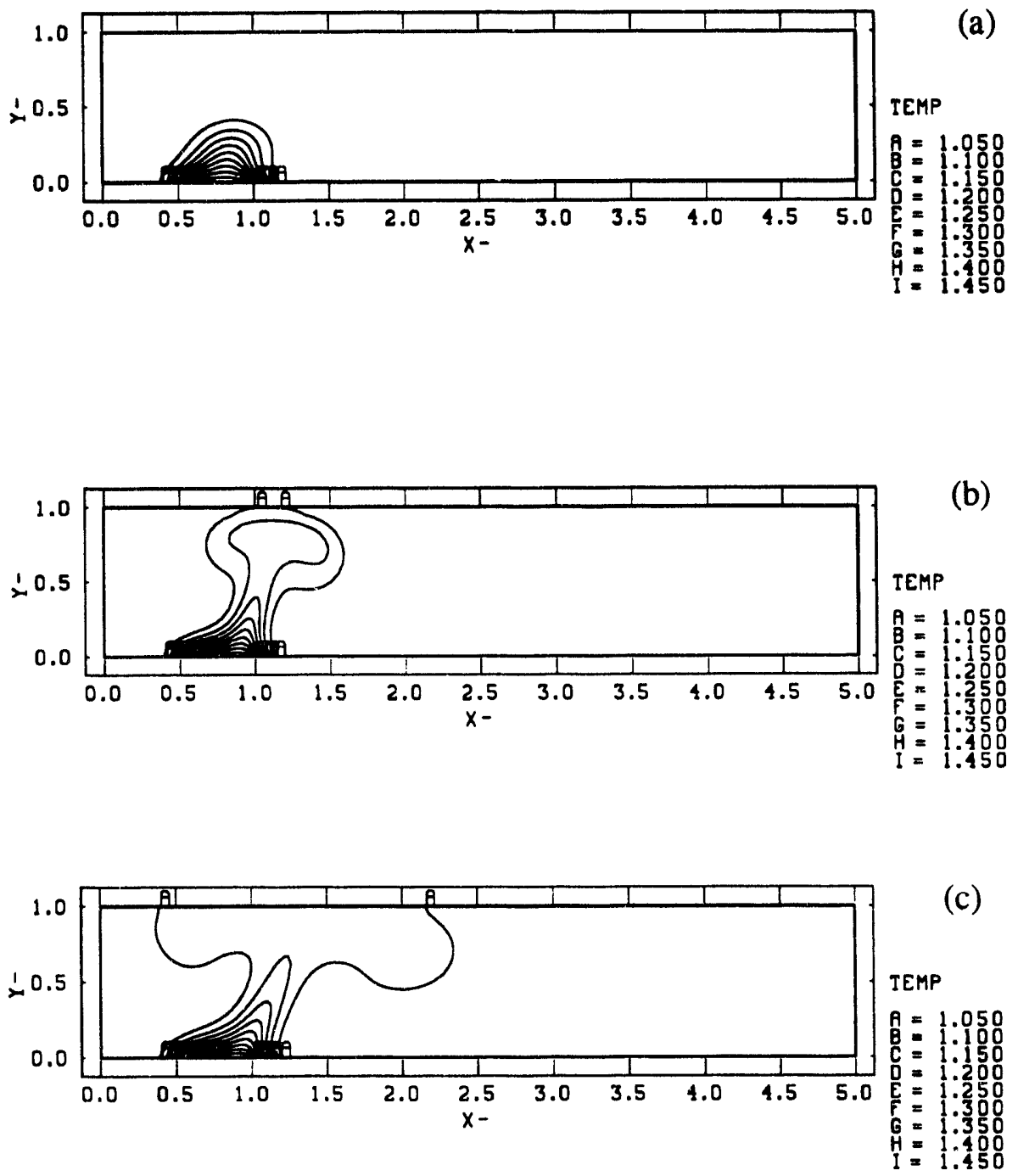

Figure 4 The evolution of temperature for the anelastic formulation, at a) $t=0.2$, b) $\mathrm{t}=0.4$, and c) $\mathrm{t}=0.6$. 


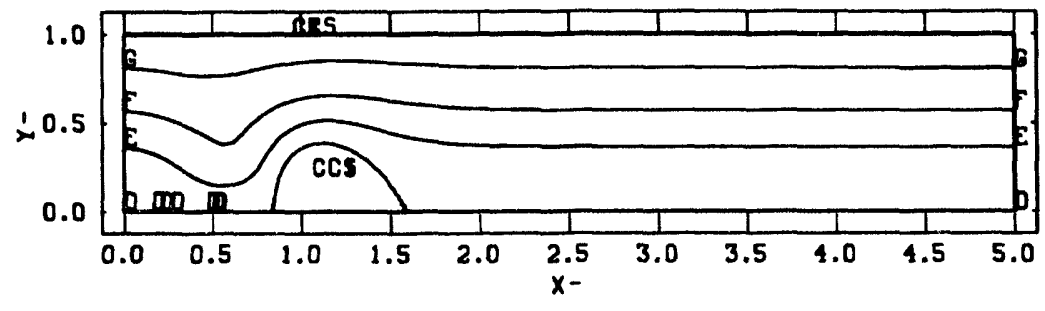

(a)

\section{STREAM}
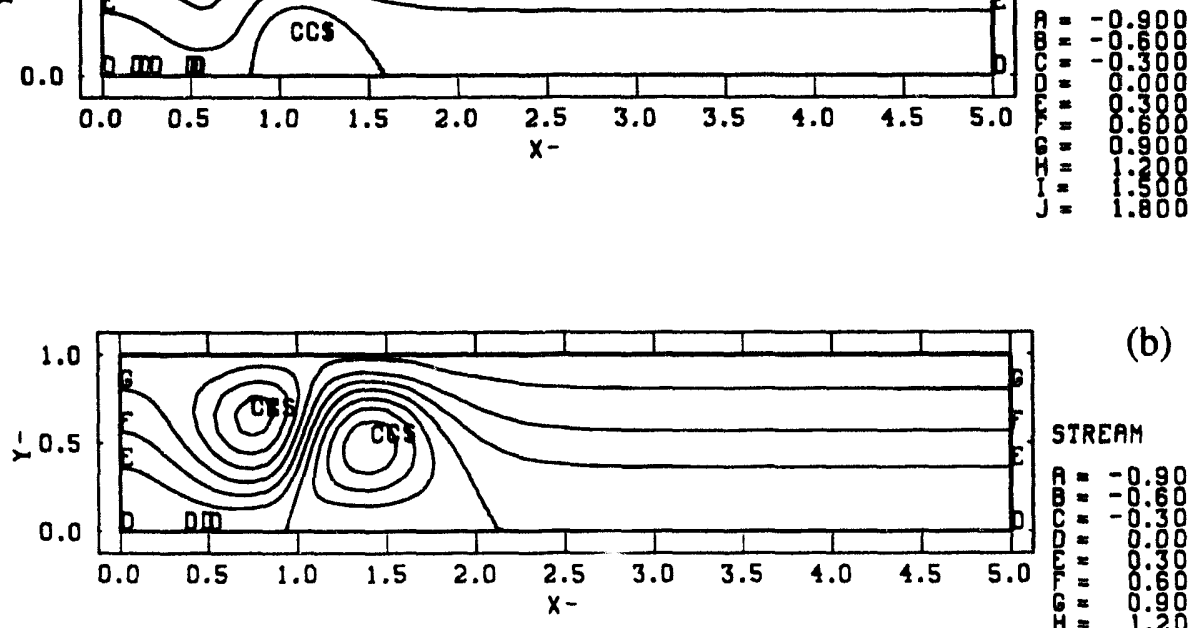

(b)
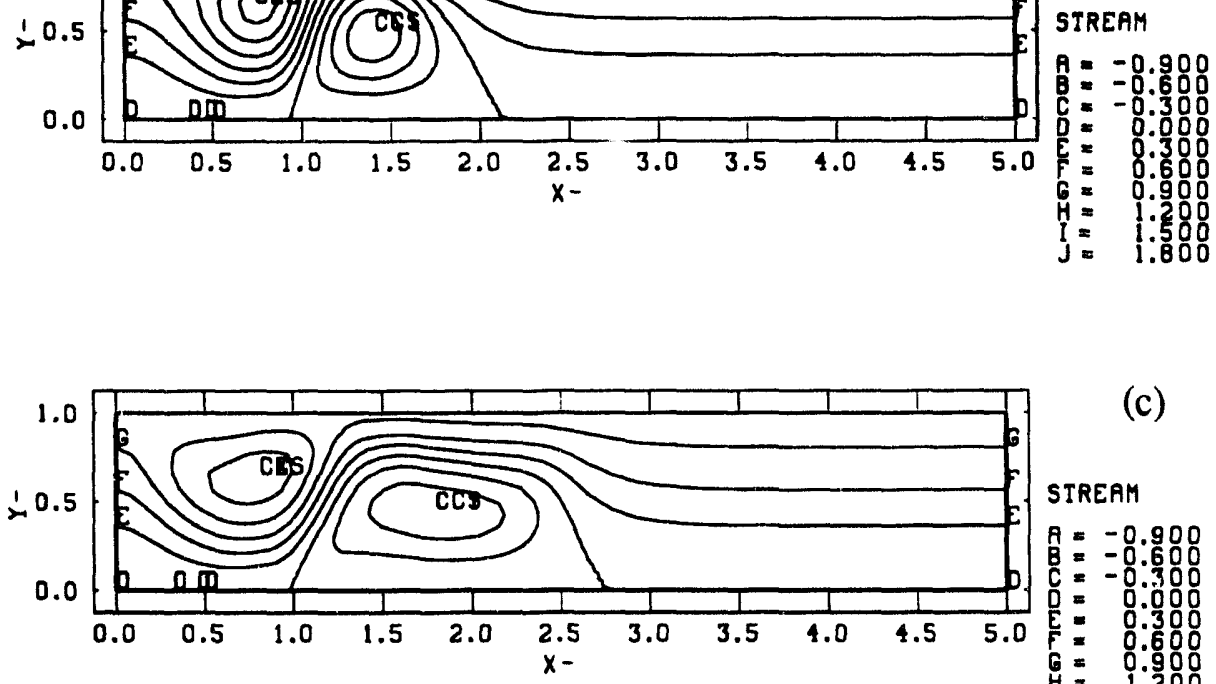

(c)

STREAM

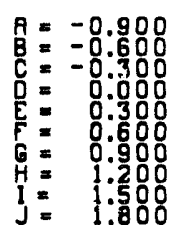

Figure 5 The evolution of the stream function for the anelastic formulation, at a) $\mathrm{t}=0.2$, b) $\mathrm{t}=0.4$, and $\mathrm{c}$ ) $\mathrm{t}=0.6$. 

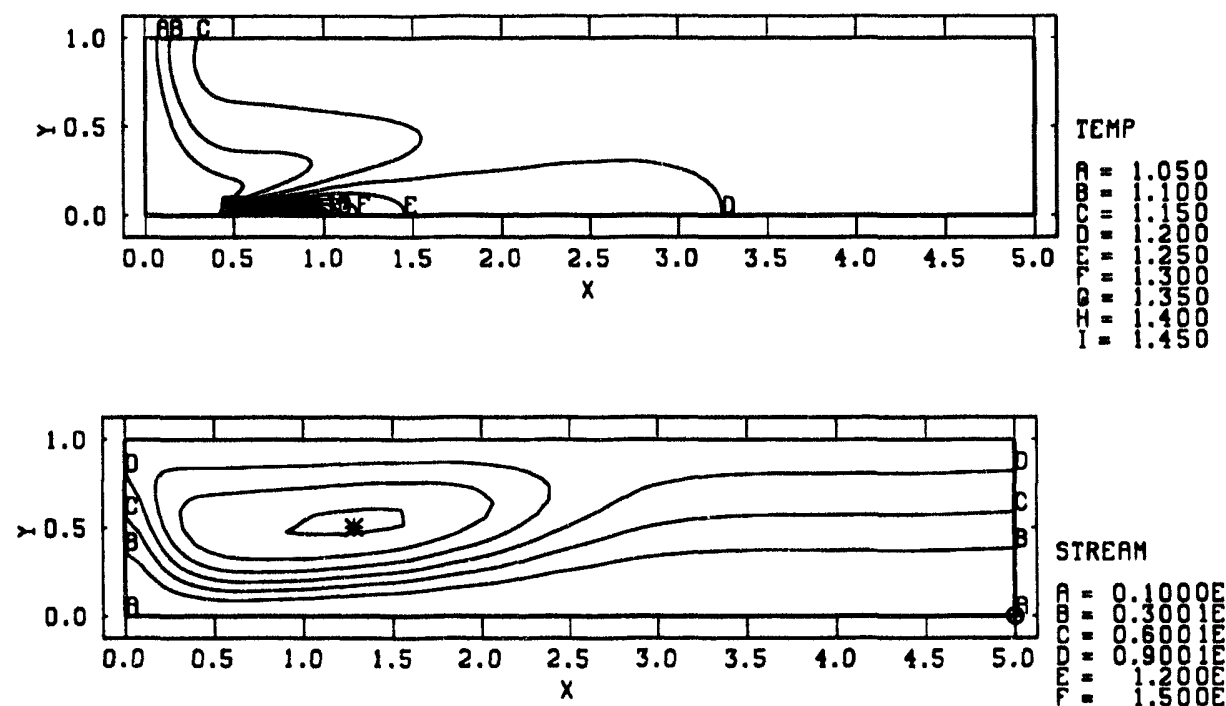

STREAM

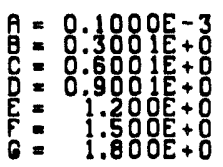

$*=-0.1001 E-3$

Figure 6 Steady distribution of temperature and stream function for the Boussinesq formulation.
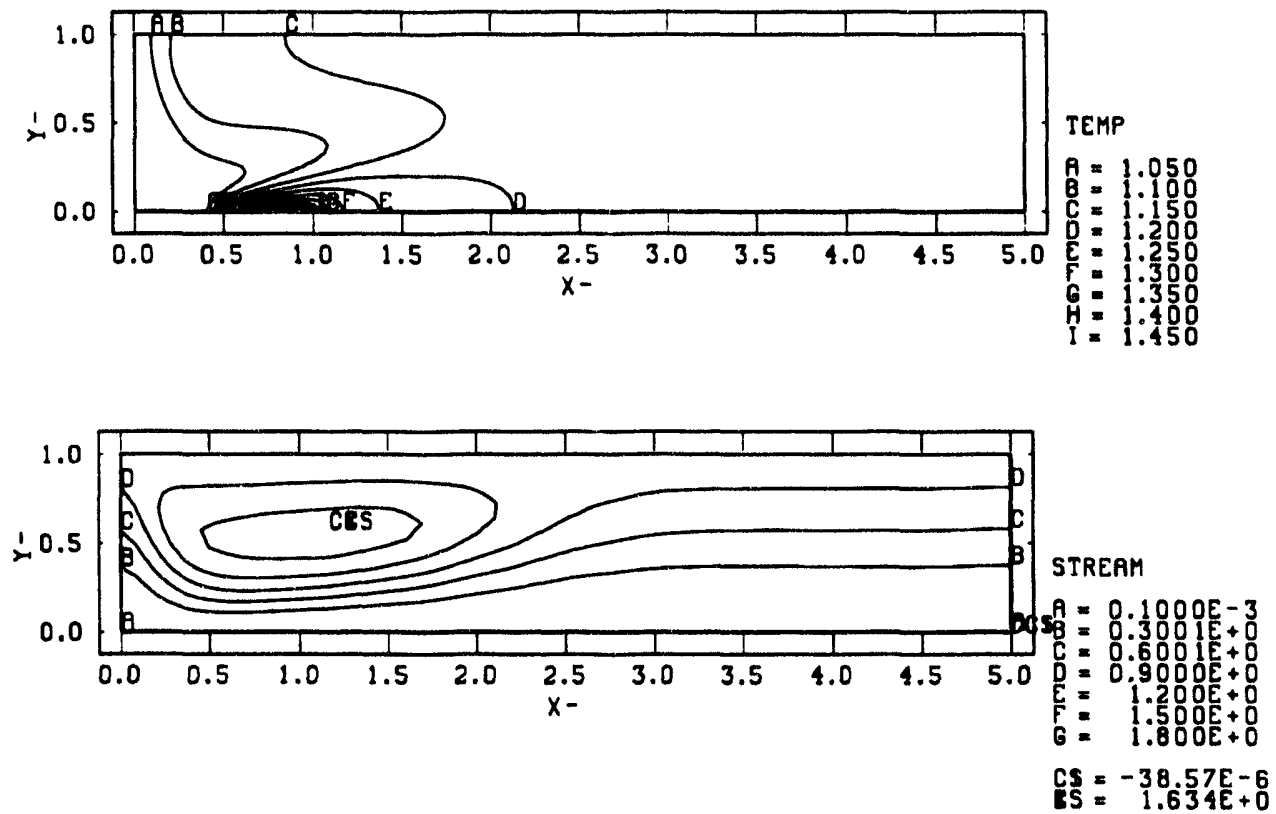

Figure 7 Steady distribution of temperature and stream function for the anelastic formulation. 
The steady distribution of temperature and stream function for the anelastic and Boussinesq approximations are shown in Figures 6 and 7. Overall, the distributions are similar, though not the same. The maximum value of the stream function, a measure of the vigor of the reverse flow cell, is larger in the Boussinesq solution. The temperature distribution is also slightly different; the BO solution is hotter downstream of the susceptor. The proximity of the susceptor to the entrance results in a noticeable temperature gradient between the entrance and the susceptor.

\subsubsection{Gas expansion and the transient evolution}

A major difference between the AF solutions and the AN and BO solutions is the treatment of the transient gas expansion caused by the heat-up of the susceptor. Upon isobaric heating of the susceptor, the surrounding gas should experience expansion. However, each formulation treats this effect differently. In the Boussinesq approximation, the assumption was made that under conditions of small temperature differences, the expansion is also small and therefore neglected. Consequently, the BO solutions display no gas expansion upon heating. The AN approximation retains a somewhat 'quasi-steady' gas expansion effect in that a general temperature-dependent density is retained, but the temporal change in density in the continuity equation is assumed of smaller order than the divergence of mass flux. Finally, the AF equations retain the full continuity equation, thereby retaining all gas expansion effects. However, because the density is independent of pressure in this approximation, acoustic effects have been eliminated. The consequences of these approximations are illustrated in Figure 8 which shows the efflux of volume at the outflow plane as a function of time for the three approximations to the equations. The influx of volume is unity for all time; the global dilatation is the excess of the volume efflux beyond unity. During the heat-up phase, $t<0.05$, there is a monotonic increase in excess volume in the AF solutions. The time at which the susceptor (which is linearly heated for $t<t_{\text {ramp }}$ ) attains its final temperature is indicated by the peak in volume efflux. There is a decrease beyond the heat-up phase until the net efflux approaches a steady value, greater than the inflow of volume, owing to the expansion of incoming gas as it passes over the susceptor. The subscripts indicate whether constant (c) or temperature-dependent variable (v) properties were specified in the anelastic simulations.

Initially, neither the $\mathrm{BO}$ nor $\mathrm{AN}$ solutions show any volume flux divergence, while the AF solution shows an instantaneous efflux of volume. Neither of these simulations is strictly correct. In reality, upon heating the susceptor, acoustic pressure and velocity waves would emanate from the region of the susceptor (Spradley and Churchill, 1975). The global divergence of volume flux would appear in the time it takes acoustic waves to reach the domain boundaries (the inflow and outflow planes). Following the acoustic waves would be a persistent divergence of volume flux owing to the heated gas inside the domain, i.e., upon heating, the same mass of gas occupies a larger volume, and hence the expansion causes a net dilatation from the region. This condition persists to steady state, if one exists, wherein the outgoing volume flowrate exceeds the incoming volume flowrate owing to the heating taking place via the susceptor. The AN solution will display this latter effect at steady state. The AN equations will only display global dilatation when the thermal is convected to the domain boundaries, prior to this there is no global dilatation. Only the AF 


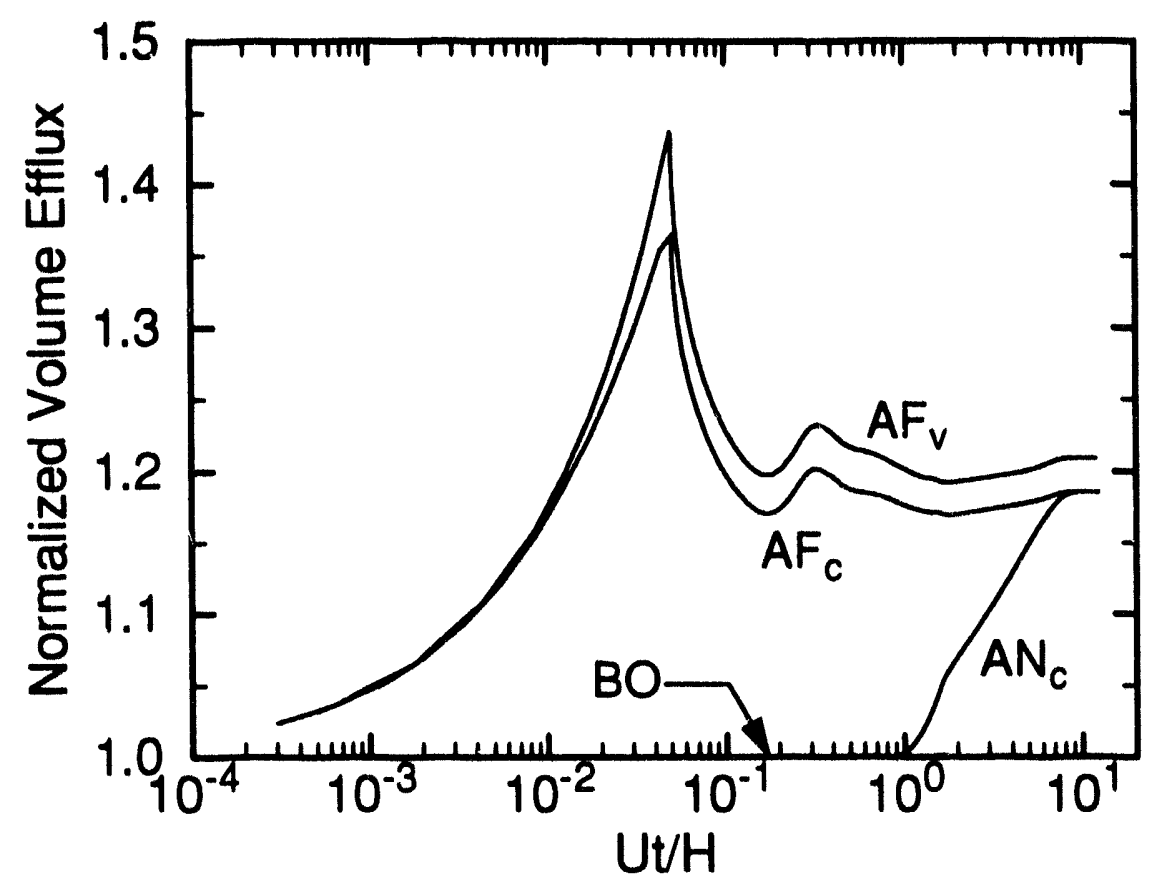

Figure 8 Volume efflux history for the three different equation formulations.

equations reproduce this transient dilatation due to gas expansion in the vicinity of the susceptor. Because acoustic effects are absent, this appears instantaneously, whereas in reality, it should appear on the order of the acoustic travel time to the boundaries. The latter is assumed to be a much shorter timescale than that of interest for problems at low Mach number, as assumed in deriving the $\mathrm{AF}$ and $\mathrm{AN}$ equations. In the example shown, the $\mathrm{BO}$ approximation underestimates the volume efflux by some $20 \%$ at steady-state, and up to $40 \%$ during the heat-up transient. Also shown in Figure 8 is the effect of constant versus temperature-dependent properties. The latter result in a larger efflux of volume throughout the simulation.

The net mass flux through the computational domain will also be nonzero initially while the gas is being heated. This effect is only retained in the AF solution as shown in Figure 9 which shows the normalized mass efflux exiting the computational domain. The BO and AN solutions show no net mass divergence at any time while the AF solutions shows the efflux of mass exceeds the influx until a steady solution is obtained. The figure indicates the steady solution is attained after about 10 units of dimensionless time have elapsed. Note that the AN solution displays no mass divergence, even though the density varies within the computational domain. This figure also serves as a mass balance check for the $\mathrm{BO}$ and $\mathrm{AN}$ solutions.

\subsubsection{Heat transfer and gas expansion}

A demonstration of Eqn (22) given earlier is shown in Figure 10 which gives the error in the computed values of this expression for the AF solution, including the case of constant $\left(\mathrm{AF}_{c}\right)$ and temperature-dependent viscosity and conductivity $\left(\mathrm{AF}_{v}\right)$. In the figure, $V$ 


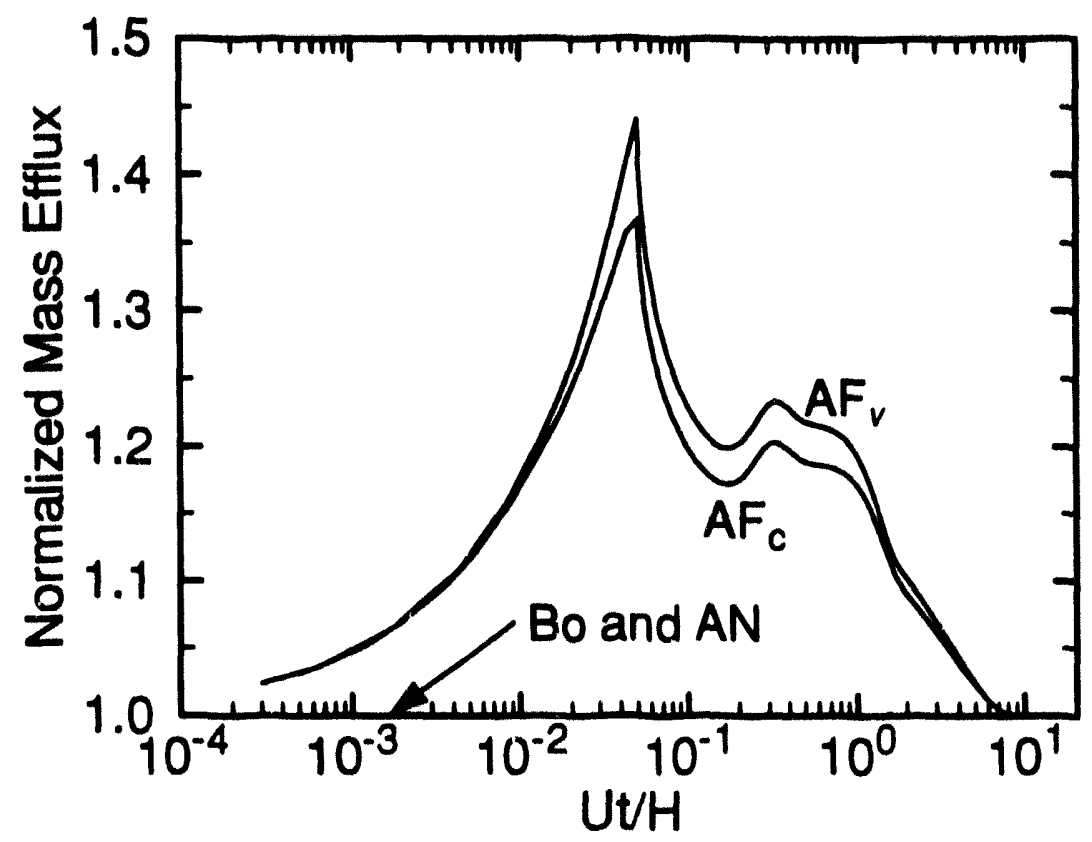

Figure 9 Mass efflux history for the three different equation formulations.

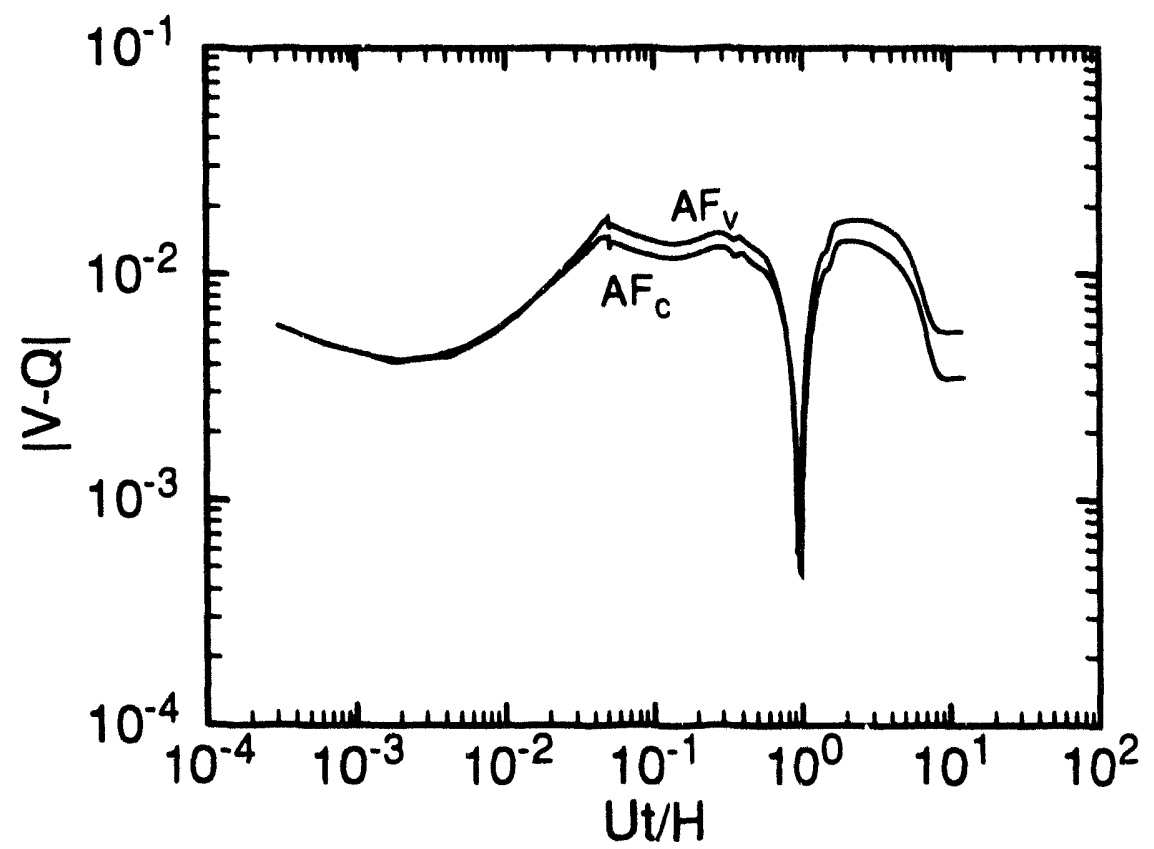

Figure 10 Error in the relation between heat transfer and the induced dilatation.

denotes the boundary integral of the volume flux and $Q$ is the boundary integral of the heat flux into the gas via the susceptor (There may be a small error incurred in neglecting the difference in conducted heat flow through the entrance and exit planes of the reactor.). We recall that (21) indicates that in the acoustically filtered approximation, the dilatation is given by the net energy transported into the fluid. Figure 10 shows that this property of the 


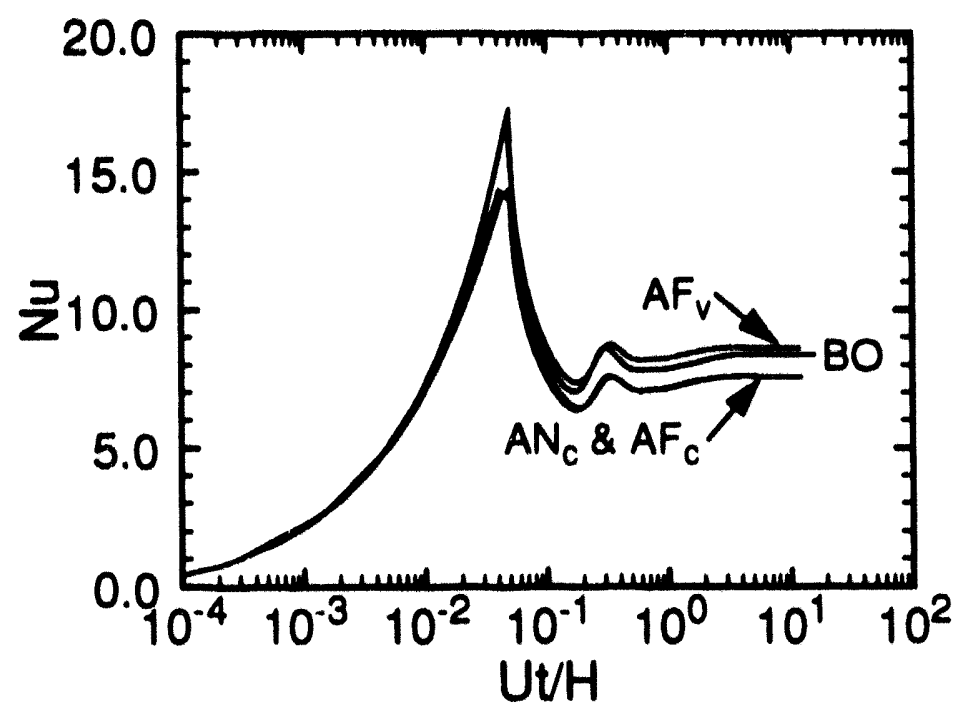

Figure 11 Nusselt number history for the three different equation formulations.

acoustically filtered equations is retained in the numerical solution, and serves as some verification of the numerical model. The comparison is reasonably good, considering we are comparing an integral of a primary variable (velocity) with an integral of the gradient of a primary variable (the heat flux); computation of the latter quantity typically results in roughly an order-of-magnitude loss of accuracy with respect to mesh spacing.

The Nusselt number averaged over the entire susceptor is shown in Figure 11 for the three different approximations to the equations. The Nusselt number is defined by

$$
N u=\frac{Q}{k_{0}\left(T_{H}-T_{0}\right)} \frac{H}{L_{s}}
$$

where $Q$ is the integral of the heat flux over the length of the susceptor, $L_{s}$, and $k_{0}$ is the conductivity evaluated at $T_{0}$ (for the variable-property AF solution). The peak value of $\mathrm{Nu}$ occurs for $t=t_{\text {ramp }}$, and decreases once the susceptor attains its final temperature. The $\mathrm{AF}_{v}$ and $\mathrm{BO}$ solutions track closely for time less than about 0.1 , while the $\mathrm{AN}_{c}$ and $A F_{c}$ solutions track closely for $t>0.1$.

At steady state the Nusselt number given by the $\mathrm{AN}_{c}$ and $\mathrm{AF}_{c}$ equations $(\mathrm{Nu} u=7.57)$ is the same (as it should be), but is less by about $9.4 \%$ than the $N u$ given by the $\mathrm{BO}$ approximation $(N u=8.36)$, and less by about $14 \%$ than the $\mathrm{AF}_{v}$ solution $(N u=8.61)$. This difference in Nusselt number is established early in the simulation and persists thereafter. Except very early in time, there is little difference in the Nusselt number histories attained with the AN and $\mathrm{AF}$ equations. It should be noted that, since constant thermophysical properties were specified, the calculations for the $\mathrm{AN}_{c}$ and $\mathrm{AF}_{r}$ equations are perhaps inconsistent. Because full density variations are allowed in the latter approximations, the temperature variation of properties can be included consistently, as they are in the $\mathrm{AF}_{v}$ solution. In con- 
trast, the assumptions leading to the BO equations, dictate that only constant properties are consistent. That is, the variable property Boussinesq equations cannot be derived through a rigorous limiting process. Nevertheless, in this case at least, the BO approximation yields a Nusselt number in closer agreement with the variable-property AF solution. The increase in heat flux for the $\mathrm{AF}_{v}$ solution can be attributed to the increase in conductivity with temperature near the susceptor. This also accounts for the increased volume efflux for the variable-property solution indicated in Figure 8.

To summarize, the AF equations display significant gas expansion effects, particularly near the susceptor during the heat-up phase. The AN and BO approximations do not display dramatic transient gas expansion features; however, the AN equations do have a persistent gas expansion effect owing to the variable density. In flowing through a temperature gradient, temperature-dependent density changes affect the fluid acceleration as well as the expansion or contraction of the fluid. These effects are preserved by the AN equations, but the flow caused by gas expansion during transient heat-up is not.

On the other hand, the expansion-induced flow during heat-up in the context of the AF approximation is the source of instantaneous accelerations everywhere in the field because acoustic effects have been removed. Consider that

$$
\int_{\Omega} \frac{\partial \rho}{\partial t} d \Omega=-\int_{\partial \Omega} \rho u \cdot n d \Gamma
$$

and in the examples shown, $\partial \rho / \partial t$ is specified at the susceptor, thereby resulting in a divergence of mass flux in the computational domain. Because at early time the flow is incompressible almost everywhere (except in the immediate vicinity of the susceptor), this mass divergence will be felt instantaneously at the exit, for example. There is also a large pressure gradient associated with the induced flow. Furthermore, the magnitude of the mass divergence and pressure gradient increases as the heat-up rate increases. We must conclude that the abruptly heated susceptor is ill-posed in this context, whereas it is allowed in the $\mathrm{BO}$ and $\mathrm{AN}$ approximations.

\subsubsection{Influence of the heating rate}

One of the more interesting differences among the numerical solutions for the various approximations to the equations is the increase in mass and volume flux exiting the channel relative to the in-coming flux. It is of interest to inquire how the rate at which the susceptor is heated to its final temperature influences the excess mass efflux during the transient. A simulation was performed in which the dimensionless rate of heating was reduced to a value of 0.1 (from a value of 10 ). This was accomplished by specifying $t_{\text {ramp }}=5$, as compared to 0.05 , the nominal value used in the preceding. Figure 12 shows the history of mass efflux out the exit plane of the channel for the two values of heating rate, and for the constant-property AF equations. Both curves show a fairly rapid efflux of mass out the channel, compared to the timescale for completing the heat-up of the susceptor, $t_{\text {ramp }}$. On an absolute timescale, the early-time efflux increases as $t_{\text {ramp }}$ decreases. Both curves also show a peak in the mass efflux for $t=t_{\text {ramp. }}$. However, the higher heating rate results in a 


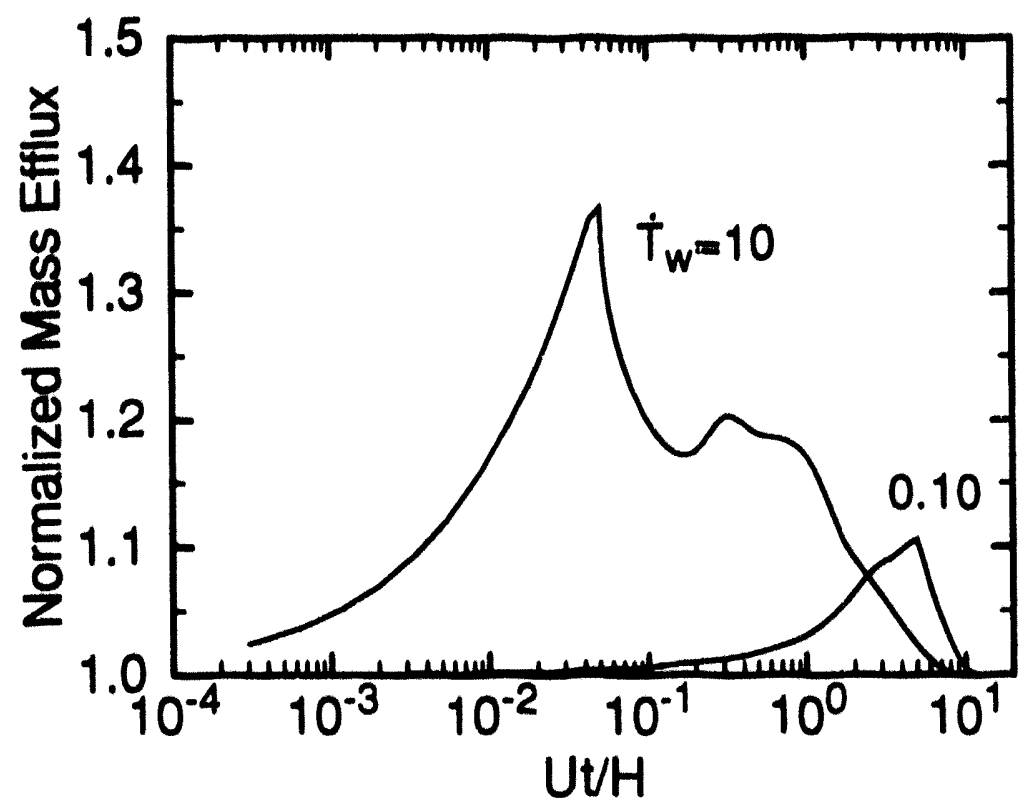

Figure 12 The influence of the heating rate on the global mass efflux from the computational domain.

larger peak mass efflux, owing to the much greater rate of heat addition. The higher the heating rate, the larger the rate of mass and volume efflux at the exit plane. The figure also implies that if the heating rate is slow enough, the expansion-induced net mass efflux vanishes, i.e., the AF approximation reverts to the AN equations for $t_{\text {ramp }}$ " $H / U$. The lower heating rate only slightly extends the time to achieve a steady-state.

\subsection{Numerical Issues}

Given that less literature exists regarding numerical simulation of low-Mach number convection with finite elements, it is perhaps worthwhile to discuss some of the numerical issues which were observed with the present scheme. This discussion is necessarily heuristic in many respects, however, it may prove useful to others interested in this class of problems.

\subsubsection{Performance of the methods}

The Newton scheme worked well, so long as all the additional sensitivities associated with the temperature-dependent density are included in all the equations. (I implemented the temperature derivatives a few at a time, and the convergence properties were observed to improve at each step.) For the AF equations, during a start-up of the time integrator, cycling of the corrector equation was necessary. That is, for the CVD problem, the first 510 timesteps required the trapezoid rule be iterated to satisfy the convergence criteria. The AN equations normally did not require cycling the corrector. Once a good start-up was attained the one-step Newton method usually worked fairly well on the AN equations. However, occasionally the auto-step strategy failed on the AF equations, and in this situation the equation cycling was essential to continue the integration. Thus, the AF equations 
are the most difficult to integrate. This is indicated by the amount of work required to integrate the equations to steady-state, nominally for $t>12$. The (constant property) BO formulation required about 290 time steps to reach $t=12$, the $A N_{v}$ required 1077 , and the constant and variable property AF equations required about 1115 time steps. However, the variable-property AF equations required iterating the corrector equation throughout much of the integration, while the constant-property AF equations only occasionally required cycling of the corrector.

In an attempt to minimize the total numiber of "function evaluations" for the time integrator, the solver strategy was first written to attempt a time step restart in preference to cycling the corrector. The latter being attempted only if the truncation error estimate indicates the predictor and corrector solutions are too far apart, defined such that the timestepping algorithm would reduce the time step to less than one-half the current value. This strategy worked fairly well for the AN equations. For high heat-up rate problems and the AF formulation, cycling the corrector equation appeared to be the better strategy, together with careful attention to start-up of the time integrator.

\subsubsection{The AF formulations}

The $\operatorname{AF}(T)$ and $\operatorname{AF}(\rho)$ formulations result in the same solutions (as they should), i.e., there is not much discernible difference in the resulting numerical solutions. The $A F(\rho)$ formulation is somewhat easier to code, especially if an analytical Newton scheme is employed. The AF(T) scheme requires the $\boldsymbol{R}^{\prime \prime}$ matrix (see the Appendix), which involves second derivatives of $\rho(T)$, requiring the added burden of computing these values in the userspecified subroutines. However, the $A F(T)$ method appears to be slightly more robust, relative to some pressure vibrations discussed in the following.

\subsubsection{Mesh-dependent pressure fluctuations}

The simulations for the CVD reactor were computed on two different finite element discretizations. Mesh 1 consisted of 400 (40x10) biquadratic elements (bilinear for pressure), while mesh 2 consisted of 825 (55x15) such elements (Results reported earlier were computed on mesh 2.). Mesh 1 was graded along the direction normal to the susceptor, with finer spacing near the susceptor, and largely uniform spacing in the streamwise direction. In mesh 2 the elements were graded toward the susceptor, both normal to and towards the fore/aft edges of the susceptor. The mesh spacing was decreased by about a factor of 4 between mesh 1 and 2 along the direction normal to the susceptor. The simulation was first performed on mesh 1, for which it was noted that use of the Adams Bashforth/trapezoid rule ( $A B / T R$ ) integrator in the AF formulation resulted in "ringing" of the pressure solution. That is, a time history of pressure at a node displays oscillations about a mean value with each timestep. The ringing was exacerbated with increasing heat-up rates, and "disappeared" as the heat-up rate decreased. Both AF formulations were prone to the ringing on mesh 1 , however, the $\operatorname{AF}(\rho)$ formulation appeared to be the more easily excited of the two. Use of the dissipative backward Euler integrator on mesh 1 eliminated the ringing. However, the same simulation on mesh 2 using the AB/TR time integrator displayed no ringing of the pressure, and in fact gave the mean-value pressure solution noted on mesh 1. Also, no such ringing was observed on either mesh with the $\mathrm{AN}$ formulation, thus one 
must conclude that the $\partial \rho / \partial t$ term in the continuity equation is the culprit. The lesson appears to be that some care is required in the mesh specification with the AF formulation, particularly in view of the fact that the heat transfer drives the time-dependent gas expansion.

\subsubsection{Basis for the pressure}

Both the bilinear and discontinuous linear element were employed. The solutions for the CVD problem were essentially the same for the two elements. The performance of the time integrator was better with the bilinear element.

\section{Concluding Remarks}

The Boussinesq and anelastic approximations were reviewed and analyzed to clarify the relationship among the various approximations. A finite element formulation for the classical anelastic and acoustically filtered approximations was presented and implemented numerically. The applications illustrated the following points:

1. The Boussinesq equations neglect all gas expansion entirely, nevertheless these equations yield relatively good heat transfer rates (at least for $\varepsilon \leq 0.5$ ).

2. For transient problems, the anelastic equations (AN) are useful for low heating rates. The acoustically filtered and anelastic approximations converge as the heating rate becomes much smaller than the characteristic flow timescale.

3. The acoustically filtered equations produce instantaneous gas expansion-induced pressures and velocities for times less than the acoustic timescale. However, if one is really interested in features on this time scale, then the fully compressible equations are appropriate. Otherwise, the AF equations are appropriate for low Mach number convection with large temperature gradients. 


\section{References}

Baum, H. R., Rehm, R. G., Barnett, P. D., and Corley, D. M., 1983, Finite difference calculations of buoyant convection in an enclosure, I. The basic algorithm, SIAM J. Sci. Stat. Comput., 4(1), 117-135.

Baum, H. R., and Rehm, R. G., 1984, Calculations of three dimensional buoyant plumes in enclosures, Comb. Sci. and Tech., 40, 55-77.

Casulli, V. and Greenspan, D., 1984, Pressure method for the numerical solution of transient, compressible fluid flows, Int. J. Num. Meth. Fluids, 4, 1001-1012.

Chenoweth, D. R., and Paolucci, S., 1986, Natural convection in an enclosed vertical air layer with large horizontal temperature differences, J. Fluid Mech., 169, 173-210.

Evans, G., and Greif, R. 1990, Unsteady convection in channel flows with applications to chemical vapor deposition, SAND89-8573, Sandia National Laboratories, Albuquerque, NM, 27 pp.

Gartling, D. K., 1987, NACHOS II -- A finite element computer program for incompressible flow problems, Part I - Theoretical background, SAND86-1816, Sandia National Laboratories, Albuquerque, $\mathrm{NM}, 70 \mathrm{pp}$.

Gresho, P. M., Lee, R. L., and Sani, R. L., 1980, On the time-dependent solution of the incompressible Navier-Stokes equations in two and three dimensions, Recent Advances in Numerical Methods in Fluids, Volume 1, Pineridge Press Ltd. Swansea, U. K., 27-81.

Gough, D. O., 1969, The anelastic approximation for thermal convection, J. Atmos Sci., 26, 448-456.

Huebner, K. H., and Thornton, E. A., 1982, The Finite Element Method for Engineers, John Wiley and Sons, New York.

Jarvis, G. T., and McKenzie, D. P., 1980, Convection in a compressible fluid with inifinite Prandtl number, J. Fluid Mech., 96(3), 515-583.

Jensen, K. V., 1987, Micro-reaction engineering applications of reaction engineering to the processing of electronic and photonic materials, Chem. Eng. Sci., 42(5), 923-958.

Karki, K. C., and Patankar, S. V., 1988, A pressure based calculation procedure for viscous flows at all speeds in arbitrary configurations, AIAA paper no. 88-0058, AIAA 26th Aerospace Sciences Meeting, Reno, Nevada.

Le Quere, P., Humphrey, J. A. C., Sherman, F. S., 1981, Numerical calculation of thermally driven two-dimensional unsteady laminar flow in cavities of rectangular cross section, Num. Heat.Trans. 4, 249-283. 
Majda, A., and Sethian, J., 1985, The derivation and numerical solution of the equations for zero Mach number combustion, Combust. Sci. and Tech., 42, 185-205.

Mueller, A. C., 1992, A preconditioned conjugate gradient Navier-Stokes solver for unstructured grids," submitted to International Journal for Numerical Methods in Fluids.

Ogura, Y., and Phillips, N. A., 1962, Scale analysis of deep and shalloiv convection in the atmosphere, J. Atmos. Sci., 19, 173-179.

Oran, E. S., and Boris, J. P., 1987, Numerical Simulation of Reactive Flow, Elsevier, New York.

Paolucci, S., 1982, On the filtering of sound from the Navier-Stokes equations, SAND828257, Sandia National Laboratories, Albuquerque, New Mexico.

Paolucci, S., 1990, Direct numerical simulation of two-dimensional turbulent natural convection in an enclosed cavity, J. Fluid Mech., 215, 229-262.

Patnaik, G., Guirguis, R. H., Boris, J. P., and Oran, E. S., 1986, A barely implicit correction for flux-corrected transport, Naval Research Laboratory Report 5855, Washington D.C.

Rehm, R. G., and Baum, H. R., 1978, The equations of motion for thermally driven, buoyant flows, J. of Res. (NBS), 83(3), 297-308.

Sherman, F. S., 1990, Viscous Flow, McGraw-Hill, Inc., New York.

Spiegel, E. A., and Veronis, G., 1960, On the Boussinesq approximation for a compressible fluid, Astrophys. J., 131, 442-447.

Spradley, L. W., and Churchill, S. W., 1975, Pressure- and buoyancy-driven thermal convection in a rectangular enclosure, J Fluid Mech., 70, 705-720.

White, F. M., 1974, Viscous Fluid Flow, McGraw-Hill, Inc., New York. 


\section{APPENDIX}

\section{Newton Iteration}

The nonlinear system Eqn. (41) is solved by Newton iteration. If each row of (41) is written as a residual equation, the system can be written in the form $R(V)=0$, with $\boldsymbol{R}=\left[\begin{array}{llll}\boldsymbol{R}_{u_{1}} & \boldsymbol{R}_{u_{2}} & \boldsymbol{R}_{\boldsymbol{P}} & \boldsymbol{R}_{T}\end{array}\right]^{T}$, and $\boldsymbol{V}=\left[\begin{array}{llll}u_{1} & u_{2} & \boldsymbol{P} & \boldsymbol{T}\end{array}\right]^{T}$, then the Newton system is

$$
J\left(V^{k}\right)\left(V^{k+1}-V^{k}\right)=-R\left(V^{k}\right),
$$

where the superscript indicates the iteration index. The Jacobian is defined by

$$
J=\left[\begin{array}{llll}
\frac{\partial R_{u_{1}}}{\partial u_{1}} & \frac{\partial R_{u_{1}}}{\partial u_{2}} & \frac{\partial R_{u_{1}}}{\partial P} & \frac{\partial R_{u_{1}}}{\partial T} \\
\frac{\partial R_{u_{2}}}{\partial u_{1}} & \frac{\partial R_{u_{2}}}{\partial u_{2}} & \frac{\partial R_{u_{2}}}{\partial P} & \frac{\partial R_{u_{2}}}{\partial T} \\
\frac{\partial R_{P}}{\partial u_{1}} & \frac{\partial R_{P}}{\partial u_{2}} & \frac{\partial R_{P}}{\partial P} & \frac{\partial R_{P}}{\partial T} \\
\frac{\partial R_{T}}{\partial u_{1}} & \frac{\partial R_{T}}{\partial u_{2}} & \frac{\partial R_{T}}{\partial P} & \frac{\partial R_{T}}{\partial T}
\end{array}\right]
$$

Entries in $J$ are computed using the definitions of the residuals, and the following matrices are defined in Eqns. (27) through (34),

$$
\begin{gathered}
\frac{\partial \boldsymbol{R}_{u_{1}}}{\partial u_{1}}=\frac{2}{\Delta t} M+C_{1}\left(R u_{1}\right)+C_{2}\left(R u_{2}\right)+C_{1}(R \mathbf{1})+\frac{4}{3} K_{11}+K_{22} \\
\frac{\partial R_{u_{1}}}{\partial u_{2}}=C_{2}(R \mathbf{1}) u_{1}+K_{21}-\frac{2}{3} K_{12} \\
\frac{\partial \boldsymbol{R}_{u_{1}}}{\partial \boldsymbol{P}}=-Q_{1}
\end{gathered}
$$

$$
\frac{\partial \boldsymbol{R}_{u_{1}}}{\partial T}=\frac{2}{\Delta t} \frac{1}{N_{u}}\left(\boldsymbol{R}^{\prime} \mathbf{1}\right)\left(B u_{1}\right)^{T}+C_{1}^{\prime}\left(u_{1}\right)+C_{2}^{\prime}\left(u_{1}\right)+g_{1} B R^{\prime}
$$




$$
\begin{aligned}
& \frac{\partial \boldsymbol{R}_{u_{2}}}{\partial u_{1}}=C_{1}(\boldsymbol{R} 1) u_{2}+K_{21}-\frac{2}{3} K_{21} \\
& \frac{\partial \boldsymbol{R}_{\boldsymbol{u}_{2}}}{\partial \boldsymbol{u}_{2}}=\frac{2}{\Delta t} M+C_{1}\left(R u_{1}\right)+C_{2}\left(R u_{2}\right)+C_{2}(R \mathbf{R})+K_{11}+\frac{4}{3} K_{22} \\
& \frac{\partial \boldsymbol{R}_{\boldsymbol{u}_{2}}}{\partial \boldsymbol{P}}=-\boldsymbol{Q}_{2} \\
& \frac{\partial \boldsymbol{R}_{u_{2}}}{\partial \boldsymbol{T}}=\frac{2}{\Delta t} \frac{1}{N_{u}}\left(\boldsymbol{R}^{\prime} \mathbf{1}\right)\left(B u_{2}\right)^{T}+C_{1}^{\prime}\left(\boldsymbol{u}_{2}\right)+C_{2}^{\prime}\left(u_{2}\right)+g_{2} B R^{\prime} \\
& \frac{\partial \boldsymbol{R}_{\boldsymbol{P}}}{\partial \boldsymbol{u}_{1}}=-\boldsymbol{Q}_{1}^{T} \boldsymbol{R} \\
& \frac{\partial \boldsymbol{R}_{\boldsymbol{P}}}{\partial \boldsymbol{u}_{2}}=-\boldsymbol{Q}_{2}{ }^{T} \boldsymbol{R} \\
& \frac{\partial R_{P}}{\partial P}=0 \\
& \frac{\partial R_{T}}{\partial u_{1}}=D_{1}(R 1) T \\
& \frac{\partial R_{T}}{\partial u_{2}}=D_{2}(R \mathbf{1}) T \\
& \frac{\partial \boldsymbol{R}_{T}}{\partial \boldsymbol{P}}=0 \\
& \frac{\partial R_{T}}{\partial T}=\frac{2}{\Delta t}\left(N+\frac{1}{N_{u}}\left(R^{\prime} \mathbf{1}\right)(B T)^{T}\right)+D_{1}\left(R u_{1}\right)+D_{1}^{\prime}(T)+ \\
& D_{2}\left(R u_{2}\right)+D_{2}^{\prime}(T)+L_{11}+L_{22}
\end{aligned}
$$

where, for example,

$$
C_{i}^{\prime}\left(u_{j}\right)=\int_{\Omega} \Phi\left(R^{\prime}\left(u_{i}\right) \Phi\right) \frac{T \Phi^{T}}{\partial x_{i}} u_{j} d \Omega
$$

with a similar definition for $D^{\prime}(T)$, and

$$
\boldsymbol{R}^{\prime}\left(\boldsymbol{u}_{i}\right)=\operatorname{diag}\left[\frac{\partial \rho}{\partial T}\right] \operatorname{diag}\left[\boldsymbol{u}_{i}\right]
$$


which can be defined in terms of the thermal expansion coefficient. Also, $R^{\prime}=R^{\prime}(1), 1$ denotes the $N_{u}$-vector in which each component is unity, and $N_{u}$ is the length of the $\Phi$. Finally,

$$
\boldsymbol{B}=\int_{\Omega} \Phi \Phi^{T} d \Omega
$$

The term $\partial \boldsymbol{R}_{\boldsymbol{P}} / \partial \boldsymbol{T}$ depends on the particular treatment of the time-dependent term in the continuity equation, as was discussed earlier. For the formulation designated $\operatorname{AF}(\rho)$, this term is given by,

$$
\frac{\partial \boldsymbol{R}_{\boldsymbol{P}}}{\partial T}=-\boldsymbol{Q}_{i}^{T}\left(\boldsymbol{R}^{\prime}\left(u_{i}\right)\right)-\frac{\eta}{\Delta t} \boldsymbol{H} \boldsymbol{R}^{\prime}
$$

where

$$
\boldsymbol{Q}_{i}^{T}\left(\boldsymbol{R}^{\prime}\left(u_{i}\right)\right)=\int_{\Omega} \Psi\left(\boldsymbol{R}^{\prime}\left(u_{i}\right) \frac{\partial \Phi}{\partial x_{i}}\right)^{T} d \Omega
$$

Thus, while this formulation, as indicated in eqn. (42), appears to move the temperature dependent density to the RHS, the Newton implementation takes explicit account of this nonlinearity in the iteration. The AF(T) formulation is somewhat more complicated, because the time-dependent density term is converted to be proportional to the temperature vector at the outset. Accounting for all temperature dependencies, this formulation results in

$$
\frac{\partial \boldsymbol{R}_{\boldsymbol{P}}}{\partial \boldsymbol{T}}=-\boldsymbol{Q}_{i}^{T}\left(\boldsymbol{R}^{\prime}\left(\boldsymbol{u}_{i}\right)\right)-\frac{\eta}{\Delta t} \boldsymbol{H}\left(\boldsymbol{R}^{\prime}+\boldsymbol{R}^{\prime \prime}(T)\right)+\frac{\eta}{\Delta t} \boldsymbol{H} \boldsymbol{R}^{\prime \prime} \boldsymbol{T}_{n}+(\eta-1) H \boldsymbol{R}^{\prime \prime} \dot{\boldsymbol{T}}_{n}
$$

where,

$$
\boldsymbol{R}^{\prime \prime}(T)=\operatorname{diag}\left[\frac{\partial^{2} \rho}{\partial T^{2}}\right] \operatorname{diag}[\boldsymbol{T}]
$$

and $R^{\prime \prime}=R^{\prime \prime}(1)$. This formulation requires two derivatives of the density with respect to temperature. 
DISTRIBUTION:

Martha Cyr

Dept. of Mechanical Engineering

Worcester Polytechnic Institute

Worcester, MA 01609

Stephen M. Cox

Dept. of Applied Mathematics

University of Adelaide

Adelaide, 5005

Australia

S. Paolucci

Dept. of Aerospace \&

Mechanical Engineering

University of Notre Dame

Notre Dame, IN 46556-9956

2 Lawrence Livermore National Laboratory

Attn: A. Almgren L-316

P. M. Gresho

P. O. Box 808

Livermore, CA 94550

P. J. Roache

Ecodynamics Research Assoc.

P. O. Box 8172

Albuquerque, NM 87198

5 University of California

Attn: G. M. Corcos

R. Grief

J. A. C. Humphrey

F. S. Sherman

K. S. Udell

Dept. of Mechanical Engineering

Berkeley, CA 94720

University of Iowa

Attn: P. B. Butler

Dept. of Mechanical Engineering

2208 Engineering Building

Iowa City, IA 52245
2 University of New Mexico

Attn: M. S. Ingber

R. Truman

Dept. of Mechanical Engineering

Albuquerque, NM 87131

D. F. McTigue

Dept. of Geological Sciences, AJ-20

University of Washington

Seattle, WA 98195

R. E. Kelly

Dept. of Mechanical, Aerospace, and Nuclear Engineering

University of California, Los Angeles

Los Angeles, CA 90024-1597

$\begin{array}{lll}\text { MS0601 } & 1126 & \text { H. K. Moffat } \\ \text { MS1111 } & 1421 & \text { W. J. Camp } \\ \text { MS1111 } & 1421 & \text { J. N. Shadid } \\ \text { MS0841 } & 1500 & \text { D. J. McCloskey } \\ \text { MS0836 } & 1501 & \text { C. W. Peterson }\end{array}$

Route to: 1554

MS0827 1502 P. J. Hommert

Route to: 1561,1564

MS0841 1504 E. D. Gorham

MS0827 $1511 \quad$ J. S. Rottler

MS0827 1511 F. G. Blottner

MS0827 1511 D. K. Gartling

MS0827 1511 C. E. Hickox

MS0827 1511 P. L. Hopkins

MS0827 1511 S. N. Kempka

15 MS0827 $1511 \quad$ M. J. Martinez

MS0827 1511 P. A. Sackinger

MS0827 $1511 \quad$ P. R. Schunk

MS0827 1511 J. A. Schutt

MS0834 $1512 \quad$ A. C. Ratzel

MS0834 1512 M. R. Baer

MS0834 1512 A. S. Geller

MS0834 1512 D. J. Rader

MS0834 1512 J. R. Torczynski

MS0835 1513 R. D. Skocypec 


\begin{tabular}{|c|c|c|}
\hline & & W. P. W \\
\hline & MS0833 1552 & C. E. Hailey \\
\hline & MS0826 1553 & W. L. Hermina \\
\hline & MS0826 1553 & B. F. Blackwell \\
\hline & MS0825 1554 & C. C. Wong \\
\hline & MS9043 8745 & R. J. Kee \\
\hline & MS9043 8745 & G. H. Evans \\
\hline & MS9043 8745 & S. K. Griffiths \\
\hline & MS9043 8745 & R. H. Nilson \\
\hline & MS07639614 & M. E. Larsen \\
\hline & MS0899 7141 & Technical Library \\
\hline & $\begin{array}{r}\text { MS0100 7613-2 } \\
\text { Proces }\end{array}$ & $\begin{array}{l}2 \text { Document } \\
\text { ssing for DOE/OST }\end{array}$ \\
\hline & MS0619 7151 & $\begin{array}{l}\text { Technical } \\
\text { Publications }\end{array}$ \\
\hline & MS9018 & \\
\hline
\end{tabular}



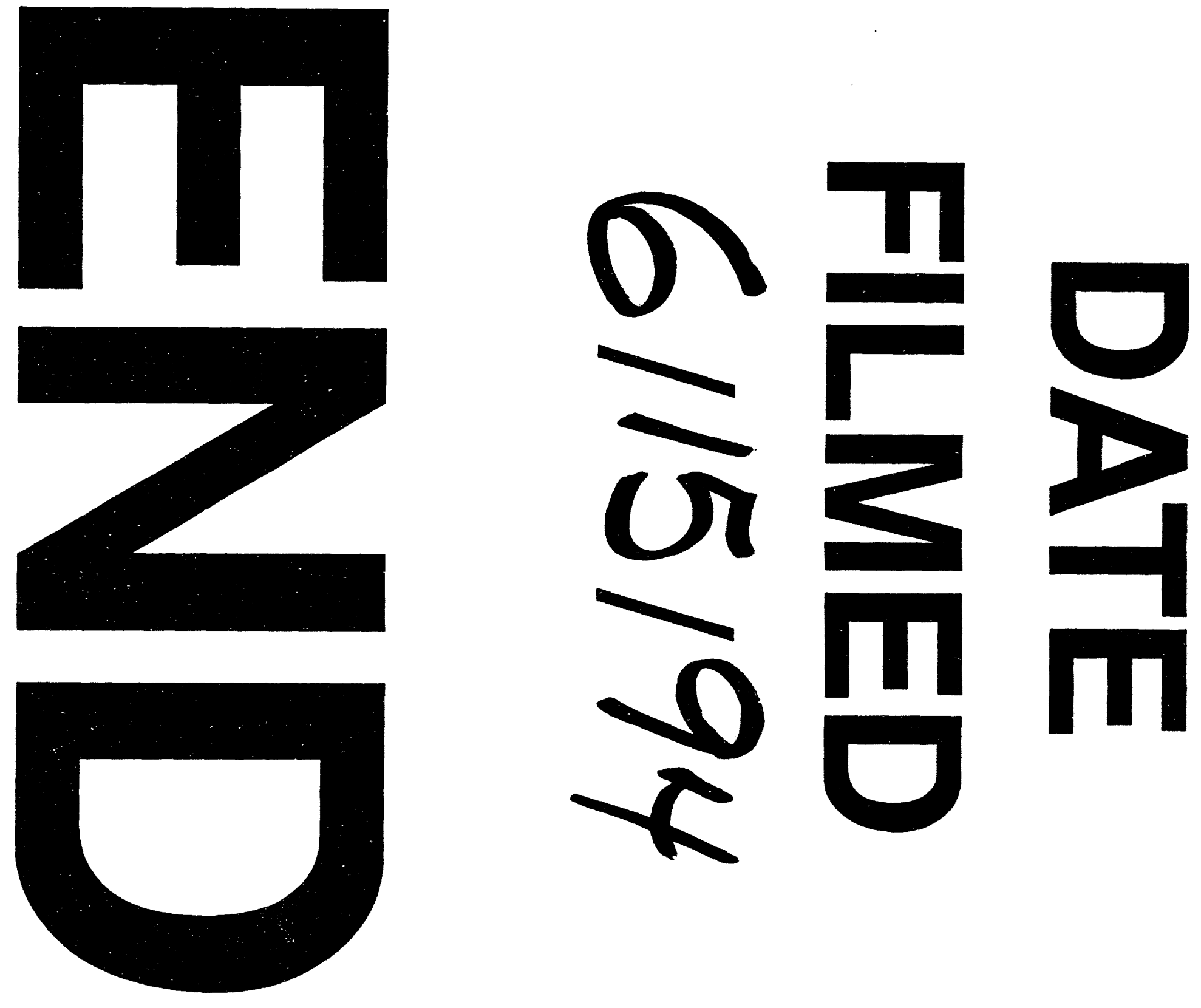
